medRxiv preprint doi: https://doi.org/10.1101/2021.11.04.21265941; this version posted November 30, 2021. The copyright holder for this preprint (which was not certified by peer review) is the author/funder, who has granted medRxiv a license to display the preprint in perpetuity.

It is made available under a CC-BY-NC-ND 4.0 International license .

1 Title- Metabolomic and lipidomic signatures in autosomal dominant and late-onset Alzheimer

2 disease brains

3

4 Authors

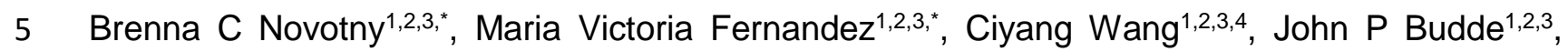
6 Kristy Bergmann ${ }^{1,2,3}$, Abdallah Eteleeb' ${ }^{1,2,3}$, Joseph Bradley ${ }^{4}$, Carol Webster ${ }^{1,2,3}$, Curtis Ebl ${ }^{1,2,3}$, 7 Joanne Norton ${ }^{1,2,3}$, Jen Gentsch ${ }^{1,2,3}$, Umber Dube ${ }^{1}$, Fengxian Wang ${ }^{1,2,3}$, John C Morris ${ }^{2,5,6}$, Randall

$8 \mathrm{~J}$ Bateman ${ }^{2,5,6}$, Richard J Perrin ${ }^{2,5,6,7}$, Eric McDade ${ }^{6}$, Chengjie Xiong ${ }^{5}$, Jasmeer Chhatwal ${ }^{8}$, 9 Dominantly Inherited Alzheimer Network Study Group, Alzheimer's Disease Neuroimaging 10 Initiative ${ }^{9}$, Alzheimer's Disease Metabolomics Consortium ${ }^{10}$, Alison Goate ${ }^{11}$, Martin Farlow ${ }^{12}$, 11 Peter Schofield ${ }^{13}$, Helena Chuil ${ }^{14}$, Celeste $M$ Karch $^{1,2,3}$, Bruno A Benitez ${ }^{1,2,3, \dagger}$, Carlos 12 Cruchaga ${ }^{1,2,3,5, \dagger}$, Oscar Harari ${ }^{1,2,3,5,+, \ddagger}$

\title{
13 Affiliations
}

$14{ }^{1}$ Department of Psychiatry, Washington University School of Medicine (WUSM), St. Louis, MO, 15 USA

$16{ }^{2}$ Hope Center for Neurological Disorders. Washington University School of Medicine, St. Louis, $17 \mathrm{MO}$, USA

$18{ }^{3}$ NeuroGenomics and Informatics Center, Washington University School of Medicine, St Louis, 19 MO, USA

$20{ }^{4}$ Division of Biology \& Biomedical Sciences, Washington University in St. Louis, St. Louis, MO, 21 USA

$22{ }^{5}$ The Charles F. and Joanne Knight Alzheimer Disease Research Center, Washington University 23 School of Medicine, St. Louis, MO, USA

$24{ }^{6}$ Department of Neurology, Washington University School of Medicine, Saint Louis, MO, USA

$25{ }^{7}$ Department of Pathology and Immunology, Washington University School of Medicine, Saint Louis, MO, USA

$27{ }^{8}$ Department of Neurology, Massachusetts General Hospital, Harvard Medical School, Boston, MA, USA

${ }^{9}$ Data used in preparation of this article were obtained from the Alzheimer's Disease Neuroimaging Initiative (ADNI) database (adni.loni.usc.edu). As such, the investigators within 
31 the ADNI contributed to the design and implementation of ADNI and/or provided data but did not

32 participate in analysis or writing of this report. A complete listing of ADNI investigators can be

33 found at: http://adni.loni.usc.edu/wp-

34 content/uploads/how_to_apply/ADNI_Acknowledgement_List.pdf

$35{ }^{10}$ Data used in preparation of this article were generated by the Alzheimer's Disease

36 Metabolomics Consortium (ADMC). As such, the investigators within the ADMC provided data

37 but did not participate in analysis or writing of this report. A complete listing of ADMC

38 investigators can be found at: https://sites.duke.edu/adnimetab/team/

$39{ }^{11}$ Icahn School of Medicine at Mount Sinai, New York, NY, USA

$40{ }^{12}$ Department of Neurology, Indiana University School of Medicine, Indianapolis, IN, USA

$41 \quad{ }^{13}$ Neuroscience Research Australia, Randwick, Sydney, NSW, Australia

$42{ }^{14}$ Neurology, Keck School of Medicine, University of Southern California, Los Angeles, CA, USA

43 * These authors contributed equally as first authors

44 † These authors contributed equally as senior authors

$46 \quad$ ‡ Corresponding author

47 Oscar Harari, PhD

48 Assistant Professor

49 Dept. of Psychiatry.

50 Washington University, School of Medicine

514444 Forest Park Ave.

52 Office 5587 - Campus Box 8134

53 St. Louis, MO, 63110

54 T: (314) 273-1862

55 Email: harario@wustl.edu 


\section{Abstract}

58 The identification of multiple genetic risk factors for Alzheimer Disease (AD) provides evidence to support that many pathways contribute to AD onset and progression. However, the metabolomic and lipidomic profiles in carriers of distinct genetic risk factors are not fully understood. The metabolome can provide a direct image of dysregulated pathways in the brain, including information on treatment targets. In this study, we interrogate the metabolomic and lipidomic signatures in the AD brain, including carriers of pathogenic variants in APP, PSEN1, and PSEN2 (autosomal dominant $A D ; A D A D), A P O E \varepsilon 4$ and TREM2 risk variant carriers, and non-carrier sporadic $A D(S A D)$. We generated metabolomic and lipidomic data from parietal cortical tissue from 366 participants with $A D$ pathology and 26 cognitively unimpaired controls using the Metabolon global metabolomics platform. We identified 133 metabolites associated with disease status (FDR $q$-value $<0.05)$. In SAD brains these include tryptophan betaine $(b=-0.57)$ and $N$ acetylputrescine $(b=-0.14)$. Metabolites associated with $S A D$ and ADAD include ergothioneine ( $b=-0.21$ and -0.26 respectively) and serotonin $(b=-0.34$ and -0.58 , respectively). TREM2 and ADAD showed association with $\alpha$-tocopherol $(b=-0.12$ and -0.12$)$ and CDP-ethanolamine $(b=-$ 0.13 and -0.10$)$. $\beta$-citrylglutamate levels are associated with SAD, ADAD, and TREM2 compared to controls $(b=-0.15 ;-0.22$; and -0.29 , respectively). Additionally, we identified a signature of 16 metabolites that is significantly altered between genetic groups ( $A A D$ vs. control $p=1.05 \times 10^{-7}$, ADAD vs. SAD $p=3.21 \times 10^{-5}$ ) and is associated with Braak tau stage and disease duration. These data are available to the scientific community through a public web browser (http://ngi.pub/Metabolomics). Our findings were replicated in an independent cohort of 327 individuals.

\section{Keywords}

Autosomal dominant Alzheimer disease, APP, PSEN1, PSEN2, APOE, TREM2, metabolomics,

81 lipidomics, $\beta$-citrylglutamate 


\section{INTRODUCTION}

Alzheimer disease $(A D)$, the most common form of dementia, is a heterogeneous and complex disease neuropathologically characterized by the accumulation of amyloid $(A \beta)$ plaques and neurofibrillary tangles in the brain. AD may develop as familial or sporadic. Recent advancements in $A D$ diagnosis and treatment could benefit from a comprehensive multi-omic approach to studying diverse biological processes, including metabolism ${ }^{1,2}$. Pathological changes in AD begin decades before the diagnosis of $A D^{3}$. Therefore, metabolomic changes linked to $A D$ pathology could precede disease onset and be highly informative for predictive models and preventative medicine. Metabolic decline is one of the first physiological changes detected in patients with mild cognitive impairment $(\mathrm{MCl})$ due to $A D^{4}$. Changes in lipid and energy metabolism are proven hallmarks of $A D$, but there are also reports of impairments in neurotransmitter, urea cycle, purine, polyamine, and bile acid metabolisms ${ }^{5}$. Current symptomatic treatments (cholinesterase inhibitors and memantine) target deficits in neurotransmitters to minimize cognitive decline ${ }^{6}$. The dysregulation of sphingolipids and glycerophospholipids in blood samples from the Alzheimer Disease Neuroimaging Initiative (ADNI) and both blood and brain samples from the Baltimore Longitudinal Study of Aging (BLSA) cohorts have been previously reported ${ }^{7-9}$. These metabolites allowed discrimination between AD and controls with high accuracy, sensitivity, and specificity ${ }^{10}$. Blood and brain endophenotype scores were then generated that summarized the relative importance of each metabolite to the severity of $A D$ pathology and disease progression. Furthermore, Stamate et al. (2019) used machine learning classifiers to demonstrate that a panel of plasma metabolites has the potential to match the area under the curve (AUC) of wellestablished cerebrospinal fluid (CSF) biomarkers when used to classify AD vs. healthy individuals ${ }^{11}$. Pathway analysis with the top 20 predictive metabolites indicated that the nitrogen pathway was overrepresented. Though much progress has been made in determining the specific metabolic changes in biospecimens from AD patients, the metabolomic landscape has yet to be fully understood.

$A D$ is highly heritable and can be caused by autosomal dominant genetic variants in the amyloid precursor protein (APP), presenilin-1 and -2 (PSEN1 and PSEN2) genes, or associated with risk factors in multiple other loci including apolipoprotein $E(A p o E)$ and triggering receptor on myeloid cells 2 (TREM2) ${ }^{12-14}$. The singularities of downstream effects of the complex AD genetic etiology are currently poorly understood. Pathogenic genetic variants in $A P P$, which is cleaved into $A \beta$ by $\beta$ - and $\gamma$-secretase, cause altered production of A $\beta$. PSEN1 and PSEN2, each crucial members of the $y$-secretase complex, can carry pathogenic variants resulting in increased cleavage of APP 
medRxiv preprint doi: https://doi.org/10.1101/2021.11.04.21265941; this version posted November 30, 2021. The copyright holder for this preprint (which was not certified by peer review) is the author/funder, who has granted medRxiv a license to display the preprint in perpetuity.

It is made available under a CC-BY-NC-ND 4.0 International license .

116 into an $A \beta$ isoform more prone to aggregation ${ }^{15}$. TREM2 interacts with $A P O E, A \beta$, and other lipids,

117 mediating the recruitment of microglia to $A \beta$ plaques ${ }^{16,17}$. Rare variants in the TREM2 gene may

118 lead to impaired microglial function, contributing to $A D$ pathology ${ }^{18}$. Apo $E$ is a critical player in

119 lipid metabolism, transport, and homeostasis in the brain, and the $\varepsilon 4$ allele of the $A P O E$ gene is

120 the main genetic risk factor for late-onset AD. Arnold et al. (2020) performed association analyses

121 of 139 serum metabolites in the ADNI cohort and observed that females carrying the APOE ع4

122 allele experience more significant impairment of mitochondrial energy production than males ${ }^{19}$.

123 These findings suggest that genetic risk factors contribute to $A D$ pathology through distinct

124 mechanisms. However, the metabolomic changes associated with AD pathology and with most

125 genetic factors are currently unknown.

126 We sought to systematically investigate the metabolic signature of AD for carriers of the major

127 AD genetic risk factors. In this study, we have interrogated the metabolomic and lipidomic

128 signatures of carriers of pathogenic variants in APP, PSEN1 or PSEN2, APOE, and TREM2 risk

129 variant carriers and compared their profiles to symptomatic AD (non-genetic), presymptomatic

130 individuals with AD neuropathological change but no or minimal decline of cognition, and

131 cognitively unimpaired controls without AD neuropathology. Our analysis uncovered common

132 profiles altered across genetically categorized brains, and metabolites and lipids specific to the 133 distinct genetic factors. 


\section{MATERIALS \& METHODS}

\section{Cohorts}

WUSM

Archived fresh-frozen post-mortem parietal cortical tissue samples were obtained from the Charles F. and Joanne Knight Alzheimer Disease Research Center Brain Bank (Knight ADRC) and the Dominantly Inherited Alzheimer Network (DIAN) at Washington University School of Medicine (WUSM). Samples were obtained with informed consent, and the study was approved by the WUSM Institutional Review Board. Data available for these samples included age at AD onset, age at death (AAD), gender, Clinical Dementia Rating® $(\mathrm{CDR} \circledast)^{20}, A P O E$ and TREM2 genotypes, ADAD variant status, and Braak stages for tau and A $\beta$. Samples were categorized based on neuropathological and genetic information: neuropathological diagnosis of $A D$ and carrier of a pathogenic variant in any of the autosomal dominant genes (APP, PSEN1, PSEN2) (autosomal dominant AD [ADAD], $\mathrm{n}=25$ ), carriers of TREM2 risk-variants (TREM2, $\mathrm{n}=21$ ), no known pathogenic variants ( $A A D, n=305$ ), no clinical symptoms (Presymptomatic, $n=15$ ), and brains with no or minimal neuropathological AD lesions identified through post-mortem neurological examination (controls [CO], $n=26$ ) (Table 1). These cohorts have been described previously ${ }^{21-29}$. One participant in the control group showed elevated tau pathology (Braak tau stage IV) but was classified as a control due to the absence of dementia (CDR 0) and lack of amyloid pathology (Braak $A \beta$ stage $A$ ). This individual's pathology is attributed to primary agerelated tauopathy $(\mathrm{PART})^{30,31}$.

Data was generated by the Duke Metabolomics and Proteomics Shared Resource, a member of the ADMC, using protocols published previously for blood samples ${ }^{19,32,33}$; a custom protocol developed for the brain samples can be found on Synapse at syn10235609. Serum (syn10235596) and DLPFC (syn10235595) data from ROSMAP quantified on the Biocrates AbsolutelDQ p180 platform were downloaded from Synapse in December 2020. The dorsolateral prefrontal cortex (DLPFC) metabolomic data from the ROSMAP studies quantified on the Metabolon Precision Metabolomics platform and preprocessed by the ADMC as described $\mathrm{in}^{34}$ were downloaded from Synapse in July 2021 (syn25878459). Details of the ROSMAP study design and methods have been described previously ${ }^{35,36}$. Disease status was determined based on a combination of 164 neuropathological and cognitive metrics. Sporadic AD was defined as individuals with a CERAD 165 assessment of "definite AD" with any Braak tau stage, or "probable AD" with Braak of at least IV. 
medRxiv preprint doi: https://doi.org/10.1101/2021.11.04.21265941; this version posted November 30, 2021. The copyright holder for this preprint (which was not certified by peer review) is the author/funder, who has granted medRxiv a license to display the preprint in perpetuity.

It is made available under a CC-BY-NC-ND 4.0 International license .

166 Controls were defined by a CERAD of "possible AD" or "not AD" with Braak less than four. All AD 167 individuals have a clinical consensus diagnosis of cognitive impairment as defined by Schneider

168 et al. (2007), and controls have a consensus diagnosis of no cognitive impairment ${ }^{37}$. From the 169 ROSMAP cohort, the following samples were analyzed: 36 SAD and 55 CO serum samples, 233 170 SAD and 94 CO DLPFC samples quantified with Metabolon, and 43 SAD and 23 CO DLPFC 171 samples quantified with Biocrates p180 (Table 1). For further analysis of the ROSMAP Metabolon 172 cohort, an additional group of $223 \mathrm{AD}$ and $154 \mathrm{CO}$ participants was considered, based on 173 consensus clinical diagnosis only (Supplementary Table 1).

$174 \quad \underline{\text { ADNI }}$

175 Data was generated by the Duke Metabolomics and Proteomics Shared Resource, a member of 176 the ADMC, using protocols published previously for blood samples ${ }^{19,32,33}$. The ADNI1 and 177 ADNIGO/2 serum metabolomic data were obtained from the ADNI database (adni.loni.usc.edu) ${ }^{33}$ 178 via the ADNIMERGE package v0.0.1 (packaged March 2018, accessed December 2020) ${ }^{38}$. The 179 ADNI was launched in 2003 as a public-private partnership led by Principal Investigator Michael 180 W. Weiner, MD. ADNI aims to test whether neuroimaging can be combined with clinical 181 assessment and other biological markers to measure the progression of mild cognitive impairment $182(\mathrm{MCl})$ and early Alzheimer disease (AD). Additional information for the ADNI studies is available 183 at www.adni-info.org ${ }^{39}$. The samples analyzed from ADNI cohorts were as follows: 184 SAD and $184224 \mathrm{CO}$ serum samples from the ADNI1 cohort, and $137 \mathrm{SAD}$ and $181 \mathrm{CO}$ serum samples from 185 the ADNIGO/2 cohort (Table 1).

187 Metabolon Precision Metabolomics ${ }^{\mathrm{TM}}$ Platform

188 Data from the Knight ADRC, DIAN, and one ROSMAP cohort were generated on the Metabolon 189 Precision Metabolomics platform. For the Knight ADRC and DIAN cohorts, 50mg frozen parietal 190 cortical tissue samples were used for metabolite quantification. Thirteen duplicate samples served 191 as technical replicates. The Metabolon Precision Metabolomics platform uses an ultrahigh 192 performance liquid chromatography-tandem mass spectrometry (UPLC-MS/MS) system 193 (Metabolon, Inc., Morrisville, USA). The platform measured 880 metabolites for the WUSM 194 dataset and 1055 metabolites for the ROSMAP dataset. These metabolites are assigned to 111 195 pathways as classified by Metabolon, known as Sub Pathways. These Sub Pathways are 196 themselves classified into nine Super Pathways: amino acids, carbohydrates, cofactors and 
medRxiv preprint doi: https://doi.org/10.1101/2021.11.04.21265941; this version posted November 30, 2021. The copyright holder for this preprint (which was not certified by peer review) is the author/funder, who has granted medRxiv a license to display the preprint in perpetuity.

It is made available under a CC-BY-NC-ND 4.0 International license .

197 vitamins, energy, lipids, nucleotides, peptides, xenobiotics, and partially characterized molecules

198 (Supplementary Table 2).

199 Biocrates AbsoluteIDQ® p180 Platform

200 The remaining ADNI and ROSMAP datasets were quantified by the Biocrates AbsolutelDQ p180

201 platform, which measures approximately 180 metabolites using a combination of ultra-high

202 pressure liquid chromatography and flow-injection analysis coupled with mass spectrometry

203 (Biocrates Life Science AG, Innsbruck, Austria). Of these 180 metabolites, 85 could be matched

204 with those quantified by Metabolon based on Human Metabolome Database Identifier (HMDB ID)

205 (Supplementary Table 2).

206 Quality Control

207 Knight ADRC and DIAN Cohorts

208 The first step in the quality control process was to verify the quantification platform's consistency

209 by evaluating the technical replicates' reproducibility. We chose the 154 metabolites with no

210 missing values and a coefficient of variation greater than 0.3 to compare between replicate pairs

211 via Pearson's correlation. Each of the 13 replicate pairs showed a correlation above 0.9,

212 demonstrating a high level of consistency. The replicate pairs were then averaged for downstream

213 analysis. For each metabolite, if only one reading was missing from a replicate pair and the non-

214 missing value was in the bottom 10\% of the metabolite's distribution, the non-missing value was

215 kept. This method assumes that the missing readings in such pairs were due to the metabolite

216 level being close to the detection limit rather than due to a technical error. Single non-missing

217 values in the top $90 \%$ of a metabolite's distribution were dropped.

218 Metabolon provided annotations for 815 of the 880 metabolites quantified analytes. The 65

219 remaining analytes were not assigned to known structural identities and were excluded from

220 further analyses. We identified 198 metabolites with missing readings in at least $20 \%$ of samples.

221 Before excluding these metabolites, Fisher's exact tests were performed to determine if any

222 showed differential missingness between SAD and CO, ADAD and CO, or ADAD and SAD. Those

223 that had significantly different missingness were tested using linear regression, corrected by AAD

224 and sex, to determine whether their non-missing readings were also significantly different in those

225 comparisons. Those metabolites that had more missing values and lower metabolite readings in

226 one status compared to another were rescued, assuming that their missingness was driven by a

227 biological effect rather than a technical artifact. In all, 10 metabolites were recovered using this

228 approach: 3-methyl-2-oxobutyrate, 4-hydroxyphenylpyruvate, acetylcholine, androsterone 
229 sulfate, cysteinylglycine disulfide, gamma-glutamyl-epsilon-lysine, gamma-

230 glutamylphenylalanine, pregnenediol sulfate, serotonin, and tryptophan betaine. The missing

231 readings for these 10 metabolites were imputed with each metabolite's respective minimum

232 reading. The 188 remaining metabolites missing at least $20 \%$ of readings were excluded from the

233 dataset (Supplementary Figure 1).

234 Raw readings were log10-transformed to better approximate a normal distribution. Outlier 235 readings (outside $1.5 \mathrm{x}$ interquartile range) for each metabolite were excluded, and the mean of 236 each metabolite's distribution was adjusted to zero.

237 After the metabolite QC, 95\% of samples were missing less than 5\% of metabolite readings; the 238 maximum missingness for a sample was $11 \%$. No samples were excluded due to the missingness 239 rate, and the remaining missing values were not imputed. Principal component analysis was then 240 performed on the scaled and imputed data provided by Metabolon to identify outlier samples using 241 the $\mathrm{R}$ function PCA from the FactoMineR package ${ }^{40}$. Four outlier samples were excluded

242 (Supplementary Figure 2). The final dataset consisted of 627 metabolites measured in 392 243 samples (Supplementary Table 3).

\section{$244 \quad$ Replication Datasets}

245 We employed the above-described procedure to perform the data cleaning and QC of the ADNI 246 and ROSMAP datasets. Briefly, replicates were averaged, removing single readings above the 247 tenth percentile of the metabolite's distribution. Metabolites without assigned structural identities 248 and metabolites missing at least $20 \%$ of readings were removed, readings were log 10 249 transformed, outlier readings were removed, and the mean of each metabolite's distribution was 250 adjusted to zero. For each dataset, samples missing greater than $20 \%$ of readings were excluded: 251 one from ROSMAP p180 brain, two from ROSMAP Metabolon brain, two from ADNI1, and one 252 from $A D N I G O / 2$. Five metabolites with missingness higher than $20 \%$ were recovered from the 253 ROSMAP Metabolon cohort according to the procedure described above, considering 254 associations with $A D$ for both the neuropathological and clinical diagnoses: saccharopine, 255 tryptophan betaine, memantine, retinol (Vitamin A) and 6-oxopiperidine-2-carboxylate. Missing 256 values for these metabolites were imputed with the metabolites' minimum readings. After QC, 257 the ADNI1 dataset included 149 metabolites in 408 samples, the ADNIGO/2 dataset included 157 258 metabolites in 318 samples, the ROSMAP serum dataset included 162 metabolites in 91 samples, 259 and the ROSMAP Metabolon brain dataset included 595 metabolites in 327 samples. The 260 ROSMAP p180 brain dataset consisted of 157 metabolites in 66 samples (Table 1). 
medRxiv preprint doi: https://doi.org/10.1101/2021.11.04.21265941; this version posted November 30, 2021. The copyright holder for this preprint (which was not certified by peer review) is the author/funder, who has granted medRxiv a license to display the preprint in perpetuity.

It is made available under a CC-BY-NC-ND 4.0 International license.

261

262

263

264

265

266

267

268

269

270

271

272

273

274

275

276

277

278

279

280

281

282

283

284

285

286

287

288

289

290

291

292

\section{Statistical analyses}

Association analyses of metabolite abundance with disease status were conducted using linear regression in $\mathrm{R}$ software version $3.6^{41}$. Metabolite levels were modeled by disease status (SAD, ADAD, and TREM2) compared to $\mathrm{CO}$, corrected by sex, AAD, and post-mortem interval (PMI). Associations with APOE $\varepsilon 4$ carrier status were also tested within the SAD status, corrected by the same variables. AAD and PMI were chosen as covariates in the model due to their correlation with the first principal component of the metabolites that passed $Q C(p<0.01)$. When comparing $A D A D$ with $C O, A A D$ was not included as it is collinear with ADAD status. Linear regression was also used to test each metabolite's association with AAD in the SAD status group, correcting for sex and PMI. The false discovery rate (FDR) was controlled using Benjamini-Hochberg correction ( $R$ function p.adjust). The $q$-value threshold for significance was established as $q<0.05$. To test differences in effect size between groups, we employed an analysis of covariance (ANCOVA) comparing the effects for metabolites in SAD and TREM2 relative to their effect in ADAD. We performed additional ANCOVA tests with individuals matched by CDR (CDR = 3), tau (Braak tau $>3$ ), and $A \beta$ (Braak $A \beta=3$ ) to test whether the differences in effect sizes were influenced by neuropathology.

We calculated the first principal component of readings for metabolites which were differentially abundant in multiple groups, similar to the "eigengene" concept in the WGCNA package ${ }^{42}$. We tested the differences in the eigenmetabolite profile between groups. We also tested for association with CDR, Braak tau stage, and disease duration, defined as the difference of age at disease onset $(A A O)$ and $A A D$. The linear regression models were corrected for sex, PMI, and AAD.

We conducted additional analyses in the ADNI and ROSMAP public metabolomics datasets to follow up on our results. For the analyses in serum, PMI was not used as a covariate as it was not applicable, and age at blood draw was used in the model rather than AAD. A meta-analysis was also performed combining the serum data from the ADNI1, ADNIGO/2, and ROSMAP datasets. The meta-analysis was carried out with the same linear model, pooling all 817 serum samples: 357 sAD and 460 CO. Meta-analysis models were additionally corrected by study.

A heatmap was constructed using Metabolon's scaled and minimum-imputed metabolite readings with the ComplexHeatmap ${ }^{43}$ and circlize ${ }^{44} \mathrm{R}$ packages. Individuals were separated into status groups (ADAD, SAD, TREM2, Presymptomatic, and CO), and the heatmap was additionally annotated with CDR and Braak tau scores for each individual. Hierarchical clustering of 
medRxiv preprint doi: https://doi.org/10.1101/2021.11.04.21265941; this version posted November 30, 2021. The copyright holder for this preprint (which was not certified by peer review) is the author/funder, who has granted medRxiv a license to display the preprint in perpetuity.

It is made available under a CC-BY-NC-ND 4.0 International license .

293 individuals was performed using the ward.D2 method in the Heatmap function from the

294 ComplexHeatmap package.

295 Pathway and network analyses were performed using MetaboAnalyst ${ }^{45,46}$ and IMPaLA pathway 296 over-representation analysis ${ }^{47}$. HMDB IDs for 105 of the 133 significant metabolites could be 297 determined and input into MetaboAnalyst and IMPaLA. MetaboAnalyst matched 103 of those IDs 298 to its database, while IMPaLA matched 74 (Supplementary Table 4). Pathways were also 299 explored in the Kyoto Encyclopedia of Genes and Genomes (KEGG) pathway database ${ }^{48-50}$. The 300 source code of the scripts employed to QC, clean and analyze the data is available at http:// 301 github.com/HarariLab/Metabolomics.

\section{Pharmacological Analysis}

303 We obtained pharmacological data for the research participants to determine any potential 304 confounding effects of medications in our analyses. Longitudinal pharmacological data were 305 available for 297 research participants from the Knight ADRC cohort. The number of time points 306 (clinical assessment dates) for each participant ranged from one to 20 visits, with a mean of 3.9. 307 The mean number of years between the most recent clinical assessment and year of death was $3082.8 \pm 2.5$, with a range of $0-14$ years. Drug group information and alternate medication names 309 were obtained from the KEGG Drug database ${ }^{48-50}$. To test for confounding medication effects, we 310 performed linear regression to test for association between medications and potentially affected 311 metabolites. We also repeated the association analyses, including only individuals who had not 312 been administered the medications within the five years preceding their death, to confirm that the 313 differential abundance was not influenced by medications.

\section{Web Browser}

315 Our results are available through a public browser at http://ngi.pub/Metabolomics. The browser was created using R Shiny version $1.4 .0^{51}$ and the shinydashboard ${ }^{52}$, shinydashboardPlus ${ }^{53}$, 317 plotly $^{54}$, DT ${ }^{55}$, shinyjs ${ }^{56}$, htmlwidgets ${ }^{57}$, RColorBrewer ${ }^{58}$, kableExtra ${ }^{59}$, and dplyr ${ }^{60} \mathrm{R}$ packages. 318 Source code is publicly available in the GitHub repository 319 http://github.com/HarariLab/Metabolomics. 
321

322

323

324

325

326

327

328

329

330

331

332

\section{RESULTS}

\section{Study Design}

In this study, we performed a metabolomics analysis of parietal cortical tissue from participants of the DIAN and Knight ADRC cohorts (Figure 1). We determined the metabolomic profile of 392 participants, including three AD genetic subgroups: autosomal dominant AD (ADAD), carriers of risk variants in TREM2 (TREM2), and sporadic AD (SAD). Detailed phenotypic information included genetic risk factors, CDR, and Braak staging for tau and A metabolites quantified, 627 passed quality control (Supplementary Table 3). We tested differential abundance using linear models adjusted for AAD, sex, and PMI and compiled the differentially abundant metabolites into a profile to distinguish between AD genetic groups. We also conducted a pathway analysis with the significantly associated metabolites. To validate our results further, we performed association analysis on a total of 393 brain samples and 817 serum samples from both the ROSMAP and ADNI cohorts.

\section{Metabolite association analysis identifies differential $\beta$-citrylglutamate levels in sporadic} AD and ADAD

Association analysis indicated that the ADAD group had the most distinct brain metabolomics profile (with 131 significant metabolites; Supplementary Table 5), whereas the profiles for TREM2 and SAD cases showed a lower number of differentially abundant metabolites, with only three ( $\alpha$-tocopherol, $\beta$-citrylglutamate, and CDP-ethanolamine) and five ( $\beta$-citrylglutamate, ergothioneine, serotonin, tryptophan betaine, and $\mathrm{N}$-acetylputrescine) significant metabolites respectively (Figure 2a-d). The Super Pathways represented in the ADAD-associated metabolites were Amino Acids (48 metabolites), Carbohydrates (12), Cofactors and Vitamins (9), Energy (2), Lipid (30), Nucleotide (12), Peptide (12), and Xenobiotics (6). We found that 99 of the 131 significant metabolites were independent of AAD (Supplementary Table 6).

Pathway analysis performed with these 131 metabolites indicated overrepresented pathways in the categories of amino acid metabolism, which accounted for the most pathways, as well as sphingolipid and vitamin metabolism (Supplementary Tables 7 and 8). We observed perturbations in several amino acid metabolism pathways, including glutamate, glutathione, tryptophan, lysine and histidine metabolisms. Perturbations in sphingolipid metabolism have been identified previously as potential biomarkers for $A D^{10}$. Altered amino acid metabolism has also been reported in multiple metabolomic studies of the AD brain ${ }^{19,32,61}$. The most notable amino acid pathways in our analysis were glutamate, glutathione, and tryptophan metabolism. Abnormal glutamate metabolism is known to cause excitotoxicity ${ }^{62}$, and alterations in glutathione 
medRxiv preprint doi: https://doi.org/10.1101/2021.11.04.21265941; this version posted November 30, 2021. The copyright holder for this preprint (which was not certified by peer review) is the author/funder, who has granted medRxiv a license to display the preprint in perpetuity.

It is made available under a CC-BY-NC-ND 4.0 International license .

354

355

356

357

358

359

360

361

362

363

364

365

366

367

368

369

370

371

372

373

374

375

376

377

378

379

380

381

382

383

384

metabolism may contribute to oxidative damage and neuronal loss ${ }^{63}$. Alterations in tryptophan metabolism, especially serotonin imbalances, have also been previously noted in the AD metabolome ${ }^{64}$. We also observed perturbations in lysine and histidine metabolism pathways and novel associations in vitamin pathways.

$\beta$-citrylglutamate (BCG) was the only metabolite significantly differentially abundant in all three genetic groups. Zhao et al. (2019) showed that serum BCG levels were significantly affected by the administration of fluoxetine, a commonly prescribed SSRI antidepressant ${ }^{65}$. In our study, five participants were documented as having taken fluoxetine at the time of their most recent clinical assessment. Five SAD participants and one presymptomatic participant had taken fluoxetine within the five years preceding their death (Supplementary Table 9). A binomial logistic regression showed that BCG levels were not associated with fluoxetine use within the five years preceding their death in individuals with $\operatorname{SAD}(p=0.98)$. Furthermore, we repeated the linear regression for SAD vs. CO, excluding the individuals who had taken fluoxetine in the past five years, and did not observe a change in the association of BCG levels between SAD and CO (effect $\left.=-0.15, q=3.7 \times 10^{-2}\right)$. AAD was not associated with lifetime usage of fluoxetine $(p=0.83)$, nor were AAO $(p=0.63)$ or disease duration $(p=0.15)$.

\section{$A P O E \varepsilon 4$ carrier status shows nominal associations with metabolites}

In an association analysis of $A P O E \varepsilon 4$ carriers vs. non-carriers in the SAD group, none of the 627 metabolites tested were significantly associated after FDR correction, though 25 metabolites were nominally significant $(p<0.05)$ (Supplementary Table 10).

\section{Follow-up in independent datasets}

We tested the differential abundance of metabolites in serum and DLPFC samples from the ADNI1, ADNIGO/2, and ROSMAP cohorts to independently validate our results. For each of these cohorts, metabolites were quantified using the Biocrates AbsoluteIDQ p180 platform, which we found to have 85 metabolites in common with the Metabolon Precision Metabolomics platform. Additionally, 379 DLPFC samples were analyzed from the ROSMAP cohort, quantified on the Metabolon platform; in this dataset, 506 metabolites were in common with the Knight ADRC cohort after QC. We identified 44 metabolites that were significantly differentially abundant with consistent effect direction in our ADAD vs. CO analysis and at least one independent dataset. Among the replicated metabolites were a-tocopherol, BCG, and serotonin (Supplementary Table 11). 
Of the seven analytes that were significant in both serum and brain cohorts, distinct direction of effect between tissue types was identified among five analytes. Specifically, 2-aminoadipate, isoleucine, valine, glutamate, and tyrosine showed a positive effect in the ADAD samples and ROSMAP SAD brains but showed a negative effect in the serum analyses. Similarly, Huo, et al. (2020) observed opposite directions of effect for glycerophospholipids between brain and serum in the ROSMAP cohort ${ }^{66}$. Serotonin and 1-linoleoyl-2-arachidonoyl-GPC showed concordant effects between the tissues, with lower abundance in ADAD and SAD than controls.

The replicated metabolites supported our previous pathway analysis findings. Components of nicotinamide metabolism (trigonelline), vitamin A metabolism (retinol/Vitamin A), and tocopherol metabolism ( $\alpha$-tocopherol/vitamin E), were replicated, supporting the role of vitamin pathways. Asparagine, methionine, threonine, and tyrosine, all part of the gamma-glutamyl cycle, were found in the replicated metabolites, along with serotonin of the tryptophan metabolism pathway. Finally, BCG, glutamate, and $\mathrm{N}$-acetyl-aspartyl-glutamate were each replicated, implicating the dysregulation of glutamate metabolism.

Like the WUSM cohort, no metabolites were significant after correction when testing metabolite associations for APOE $\varepsilon 4$ carriers vs. non-carriers. However, 23 were nominally significant $(p<$ 0.05), of which none were replicated from the WUSM cohort (Supplementary Table 10).

\section{A metabolic profile associated with AD duration and Braak stage}

We sought to investigate whether ADAD, TREM2, and SAD showed a similar or more distinct difference in their altered metabolomic profile. Thus, we selected the 17 metabolites that were significantly associated in the ADAD brains ( $q$-value $<0.05)$ that also were nominally associated in both the SAD and TREM2 when compared to controls (Table 2). Of these, ergothioneine, serotonin, BCG, CDP-ethanolamine, and a-tocopherol were statistically significant after FDR correction in SAD (ergothioneine, serotonin, and BCG) or TREM2 (BCG, CDP-ethanolamine, and a-tocopherol) (Table 2). These five metabolites showed lower abundance in the AD groups as compared to controls. This group of 17 metabolites was considered for the identification of a metabolic profile differentiating between status groups. Serotonin was excluded from the profile because the ADAD group was missing 17 of 25 readings for serotonin (68\%). In the ROSMAP Metabolon cohort, the differential abundance of nine out of the 16 remaining metabolites was replicated after FDR correction: $\mathrm{V}$-glutamylthreonine, $\beta$-citrylglutamate, glutamate, $\mathrm{N}$ acetylglutamate, 1,5-anhydroglucitol, glutarate, CDP-choline, retinol, and a-tocopherol. Additionally, aspartate, ergothioneine, 2-methylcitrate/homocitrate, and glycerophosphoinositol were nominally significant, and 3-hydroxy-2-ethylpropionate did not pass QC. 
We next compared the magnitude of the effects of the remaining 16 common metabolites across the three genetic groups. The effect in ADAD tended to be greater than that of TREM2, which was in turn greater than the effect in SAD (Figure 3B). An ANCOVA test showed that the relative effects of SAD and TREM2 to ADAD were significantly different $\left(p=4.37 \times 10^{-04}\right)$. This difference in effect was reproduced when individuals were matched by CDR $\left(p=2.54 \times 10^{-02}\right)$ as well as Braak stage for $\operatorname{Tau}\left(p=1.18 \times 10^{-03}\right)$ and $A \beta\left(p=2.20 \times 10^{-03}\right)$.

Among the 16 common metabolites across the three genetic groups, four were also associated with $A A D$ in a linear regression corrected for sex and PMI: 1,5-anhydroglucitol (1,5-AG), glycerophosphoinositol, $\mathrm{N}$-acetylglutamate, and retinol (vitamin A).

We then calculated the first principal component for these 16 metabolites to generate an "eigenmetabolite" representing the metabolic profile for each individual" ${ }^{42}$ The eigenmetabolite was found to be associated with disease duration in $\operatorname{SAD}\left(p=1.86 \times 10^{-02}\right)$, as well as Braak Tau stage $\left(p=4.17 \times 10^{-11}\right)$ and $\operatorname{CDR}\left(p=4.23 \times 10^{-13}\right)$ in the entire cohort, with a lower eigenmetabolite value being associated with longer duration, higher Braak stage, and higher CDR. Eigenmetabolite values were significantly different between status groups, with ADAD, TREM2, and SAD having significantly lower eigenmetabolite values than $C O$ and ADAD having significantly lower values than SAD (Figure 3C). To validate these observations, 15 metabolites with available data for the ROSMAP Metabolon dataset (all except 3-hydroxy-2-ethylpropionate) were used to generate an eigenmetabolite profile for the ROSMAP participants. The eigenmetabolite was again associated with disease duration $\left(p=2.68 \times 10^{-02}\right)$, but was not associated with Braak tau stage $(p$ $=0.38)$. Eigenmetabolite values were not significantly associated with $S A D$ in the neuropathological categorization $(p=0.91)$, but were associated with consensus clinical diagnosis, with lower values observed in $\operatorname{AD}$ participants $\left(p=2.73 \times 10^{-3}\right)$ (Figure 4).

To visualize levels of the 16 metabolites between status groups in the WUSM cohort, a heatmap was generated with the scaled and imputed metabolite readings from Metabolon (Figure 3A). A group of 30 sAD individuals, identified by hierarchical clustering, with metabolite abundance profiles not significantly different from the control group was selected for further analysis. For these individuals, the eigenmetabolite was not significantly different from the control group in a logistic regression correcting for $\mathrm{AAD}$, sex, and $\mathrm{PMI}(p=0.66)$. These individuals were classified as Early-Stage AD (ESAD) after further logistic regression analysis of CDR, Braak tau, and disease duration, correcting for the same variables. The ESAD group showed lower CDR (ESAD $=1.67 \pm 1.09, \mathrm{sAD}=2.56 \pm 0.84$, effect $\left.=-0.83, p=8.02 \times 10^{-6}\right)$ and Braak Tau $(\mathrm{ESAD}=4.05 \pm 1.36$, $\mathrm{SAD}=5.30 \pm 1.06$, effect $=-0.66, p=1.22 \times 10^{-4}$ ) compared to $\mathrm{SAD}$. The ESAD individuals also 
451

452

453

454

455

456

457

458

459

460

461

462

463

464

465

466

467

468

469

470

471

472

473

474

475

476

477

478

479

480

481

482

showed a shorter disease duration $(E S A D=7.97 \pm 5.33, \mathrm{sAD}=10.03 \pm 4.61$, effect $=-0.09, p=$ $4.42 \times 10^{-2}$ ) than the rest of the SAD individuals.

To explore the relationship between this metabolic profile and disease progression, presymptomatic individuals were also considered. The eigenmetabolite values for the presymptomatic group were not significantly different from the CO and ESAD groups $(p=0.18$ and $p=0.06$, respectively) (Figure $3 \mathbf{C}$ ). As expected, the presymptomatic status group showed significantly lower CDR (effect $=-7.52, p=2.47 \times 10^{-03}$ ) than the ESAD group. However, the presymptomatic group did not differ from ESAD in the Braak Tau stage $(p=0.92)$.

The super pathways associated with these 16 metabolites were mostly related to amino acid metabolism (glutamate, arginine, lysine, glutathione, histidine, tryptophan), but phospholipid and vitamin pathways were also identified. CDP-ethanolamine, CDP-choline, and glycerophosphoinositol were associated with phospholipid metabolism, while a-tocopherol (vitamin E), retinol (vitamin A), and nicotinamide (vitamin B3) were components of vitamin metabolism.

Considering that three of the metabolites in the eigenmetabolite profile were vitamins: retinol (vitamin A), a-tocopherol (vitamin E), and nicotinamide (vitamin B3), we also investigated participants who had taken vitamin supplements to rule out any confounding association. Within the five years preceding their deaths, 132 individuals took vitamin E supplements, 87 individuals took vitamin A, and 101 individuals took vitamin B3 (Supplementary Table 9). When regressions were repeated excluding individuals who took vitamin $E$ supplements, we observed that all of the associations remained significant in TREM2 $\left(p=3.9 \times 10^{-04}\right)$, ADAD $\left(p=2.9 \times 10^{-05}\right)$, and $\operatorname{sAD}(p=$ $\left.2.9 \times 10^{-04}\right)$. Similarly, excluding participants taking vitamin A supplements did not affect the association of retinol with any genetic group (ADAD $p=6.11 \times 10^{-04}, \operatorname{AD} p=1.40 \times 10^{-02}$, TREM2 $p$ $=4.75 \times 10^{-02}$ ). When participants who took vitamin B3 were excluded, ADAD and TREM2 were still nominally associated with nicotinamide (ADAD $p=9.4 \times 10^{-03}$, TREM2 $p=3.7 \times 10^{-02}$ ), however, sAD was no longer associated $(p=0.11)$. Removing nicotinamide from the eigenmetabolite did not affect the eigenmetabolite association with disease duration (effect $=-0.052, p=1.75 \times 10^{-02}$ ), $\operatorname{CDR}\left(\right.$ effect $\left.=-0.65, p=4.49 \times 10^{-14}\right)$, or Braak tau (effect $=-0.57, p=6.75 \times 10^{-12}$ ).

\section{Web Browser}

The browser facilitates exploration of our analyses and further investigation into individual metabolites by integrating metadata with visualizations of our results. The browser has two main pages, or tabs. The first displays a table including metadata on each metabolite that passed our 
medRxiv preprint doi: https://doi.org/10.1101/2021.11.04.21265941; this version posted November 30, 2021. The copyright holder for this preprint (which was not certified by peer review) is the author/funder, who has granted medRxiv a license to display the preprint in perpetuity.

It is made available under a CC-BY-NC-ND 4.0 International license .

483 QC process, along with its effect, $p$-value, and $q$-value for each comparison discussed here. The

484 table allows the user to select a metabolite, which displays the distribution of the selected

485 metabolite's readings across disease statuses. Links are also provided to the PubChem

486 (https://pubchem.ncbi.nlm.nih.gov/ $)^{67}$ and Human Metabolome Database (www.hmdb.ca) ${ }^{46,68}$

487 webpages if the IDs are available. The second tab displays volcano plots for each regression,

488 with $q$-values less than 0.05 highlighted. Again, the user may select a metabolite on the volcano

489 plot to view its reading distributions among statuses and display further information and links. 
medRxiv preprint doi: https://doi.org/10.1101/2021.11.04.21265941; this version posted November 30, 2021. The copyright holder for this preprint (which was not certified by peer review) is the author/funder, who has granted medRxiv a license to display the preprint in perpetuity.

It is made available under a CC-BY-NC-ND 4.0 International license .

490

491

492

493

494

495

496

497

498

499

500

501

502

503

504

505

506

507

508

509

510

511

512

513

514

515

516

517

518

519

520

521

522

\section{DISCUSSION}

In this study, we have performed a metabolomics analysis of parietal cortical tissue from participants of the DIAN and Knight ADRC cohorts. We have characterized the metabolomic profile of three genetically defined AD subgroups including ADAD, carriers of risk variants in TREM2, and SAD. We have analyzed the detailed phenotypic information available for these brains, including genetic risk factors and clinical, pharmacological, and neuropathological variables.

We found a significantly different metabolic profile in ADAD patients from that of healthy individuals, with 131 significant metabolites linked to ADAD, altering multiple pathways including the $\mathrm{y}$-glutamyl cycle, tRNA charging, and aminoacyl-tRNA biosynthesis (Supplementary Tables

7 and 8). The parietal cortex of ADAD individuals has been reported to have a higher burden of neurofibrillary tangles (NFT) than that of SAD individuals ${ }^{69}$. Accordingly, the metabolic profiles of TREM2 and SAD showed fewer differences than ADAD from that of healthy individuals, and of the two only SAD showed metabolite differences unique to its category (tryptophan betaine and $\mathrm{N}$-acetylputrescine). Tryptophan betaine is an $\mathrm{N}$-methylated form of tryptophan, which is the serotonin precursor and has been found de-regulated in $\mathrm{MCl}-\mathrm{AD}^{70}$. N-acetylputrescine is an acetyl-CoA-ated putrescine and a GABA precursor that was found to build up in stable $\mathrm{MCl}$ but not in $A D$, where putrescine is preferentially metabolized to other polyamines ${ }^{71}$. We also observe depleted $\mathrm{N}$-acetylputrescine levels in SAD in our data (Supplementary Figure 3) which supports previously reported findings of lower GABA levels in $A D^{72}$.

Among the metabolites identified as differentially abundant in at least one group were BCG, $\alpha$ tocopherol, and ergothioneine. Each of these metabolites showed lower concentrations in an AD subgroup compared to control. BCG acts as an iron carrier to activate aconitase activity ${ }^{73}$. We observed that BCG had the lowest abundance in ADAD, again followed by TREM2 and SAD. This observation could be associated with a lower activation of aconitase and lower energetic metabolism. BCG is also a component of glutamate metabolism, and BCG levels can be increased by the selective serotonin reuptake inhibitor (SSRI) fluoxetine ${ }^{65}$. BCG levels in our cohort were not significantly associated with fluoxetine administration, indicating that the association of BCG and $A D$ in the three genetic groups is not driven by fluoxetine usage. Within the vitamin pathway, $\alpha$-tocopherol (vitamin E) was differentially abundant in both TREM2 and $A D A D$ vs $C O$, and vitamin $E$ supplementation in participants did not affect this association. Vitamin $E$ is a powerful antioxidant that aids the immune system and keeps blood clots from forming ${ }^{74-76}$. This finding complements our observation of lower BCG in SAD cases. Reduction 
523 of aconitase activity due to oxidative stress in aging could be exacerbated in AD by lower levels

524 of antioxidants such as vitamin E. This could lead to less energetic metabolism activation overall.

525 Ergothioneine was also observed at lower levels in SAD and ADAD cases compared to controls.

526 Ergothioneine is a naturally occurring amino acid and thiourea derivative of histidine produced by

527 fungi, which has antioxidant and anti-inflammatory properties ${ }^{77,78}$. The main source of

528 ergothioneine in humans is diet; it accumulates in erythrocytes and crosses the blood-brain

529 barrier $^{79}$. However, its physiological role in humans is not known. Ergothioneine blood levels in

530 humans decline with age and decline faster in individuals with cognitive impairment compared to

531 age-matched individuals with no cognitive impairment ${ }^{80}$. In mice treated with

532 intracerebroventricular injection of $A \beta 1-40$, ergothioneine protected against loss of memory and

533 learning abilities ${ }^{81}$.

534 In addition to BCG, $\alpha$-tocopherol, and ergothioneine, we identified eight metabolites in the

535 Vitamins pathway that were significant after FDR correction in ADAD vs healthy individuals and

536 significant before correction in the AD vs CO, TREM2 vs CO, and ROSMAP brain analyses. Four

537 of these (2-aminoadipate, serotonin, tryptophan and tyrosine) were also significant in the serum

538 meta-analysis and are important neurotransmitters.

539 Neurotransmitters, especially serotonin, have been shown to play a role in processing APP and 540 reducing generation of $A \beta_{42}$ through activation of the ERK signaling cascade ${ }^{82}$. In our study, 541 serotonin levels were significantly decreased in SAD and ADAD compared to control, and 542 nominally decreased in TREM2. This effect was replicated in independent datasets of both serum 543 and DLPFC tissue. SSRIs, which increase serotonin levels in the brain, show promise for 544 reduction of $A \beta$ accumulation in both the brain and CSF. Studies in APP/PS1 transgenic mice 545 showed that the SSRI citalopram caused a 50\% reduction in brain amyloid plaque load, and 546 escitalopram, citalopram's S-isomer, reduced interstitial fluid $A \beta$ by $25 \%{ }^{82,83}$. A controlled clinical 547 trial of cognitively normal adults showed that escitalopram could decrease CSF $A \beta_{42}$ levels in 548 humans, with a difference of $11.1 \%$ between the control and treatment groups ${ }^{84}$. Our results 549 corroborate the association of low serotonin with $A D$, and suggest that this effect, and potentially 550 the benefit of serotonin modulation via SSRIs, spans all three genetic groups.

551 Our discovery dataset identified a set of 16 metabolites whose first principal component, or 552 eigenmetabolite, was distinct between the AD groups and healthy individuals, and between SAD 553 and ADAD. The effects for these metabolites were greatest in ADAD, followed by TREM2 and 554 SAD. The eigenmetabolite was additionally associated with CDR, Braak tau stage, and disease 555 duration. The association with disease duration was validated in an independent dataset using 15 
of the 16 metabolites. We also evaluated the performance of these 16 metabolites in a group of presymptomatic individuals and observed that this group showed a similar profile to that of healthy individuals. In addition, we identified a set of SAD cases (Early-Stage AD/ESAD) with a metabolic profile close to that of the healthy individuals. Further examination of these individuals revealed significantly lower CDR and Braak Tau scores than the rest of SAD individuals. The presymptomatic and ESAD groups showed no significant difference in the metabolic eigenmetabolite or Braak tau but significantly different CDR. Furthermore, ADAD individuals, known to have an earlier age at onset and higher NFT burden, showed a greater effect for these metabolites. Together, these observations suggest that the metabolic profile could be driven by tau pathology and implicated in disease duration.

Spermidine was negatively associated with age in SAD and showed increased levels in SAD in the ADNIGO/2 dataset compared to control (Supplementary Tables 5 and 6). However, we did not find it associated with any genetic group in the Knight ADRC dataset. Putrescine was significantly decreased in SAD before correction (Supplementary Table 5). N-acetylputrescine, a putrescine derivative, was significantly decreased in the SAD group compared to control and significantly decreased before FDR correction in TREM2. Schroeder, et al. (2021) found that polyamines, particularly spermidine, improved cognition in mice by enhancing mitochondrial function through hypusination of eukaryotic translation initiation factor $5 \mathrm{~A}$ (elF5A) ${ }^{85}$. Liang, et al. (2021) also showed that elF5a activity decreased with age in fly models, and that spermidine supplementation could improve mitochondrial function ${ }^{86}$.

This study identified differences in metabolite abundance both specific to and common between genetically defined AD subgroups. We replicated our main findings in three independent datasets. Differences in the levels of common metabolites allow us to generate a metabolic profile associated with disease duration, CDR, and Braak tau stage and that further identified a subset of $A D$ cases with a profile similar to $C O$ (ESAD). Metabolomics of the brain can identify metabolic signatures specific to $A D$ genetic subgroups. These metabolites may support the creation of "metabolomics scores" to assess disease status. Limitations of our study include the sample size of some of the genetic groups, e.g. TREM2. As such, in future studies we would like to increase the sample size of our brain-sourced dataset. We were unable to find associations with Braak $A \beta$ stage possibly because scores are unavailable for many participants. Direct replication of our results in ADAD individuals was unachievable due to a lack of independent ADAD datasets. Unlike previous studies, we did not find significant associations between $A P O E \varepsilon 4$ carriers and non-carriers in cases of sporadic AD. In addition, our ability to replicate findings in other tissues, 
medRxiv preprint doi: https://doi.org/10.1101/2021.11.04.21265941; this version posted November 30, 2021. The copyright holder for this preprint (which was not certified by peer review) is the author/funder, who has granted medRxiv a license to display the preprint in perpetuity.

It is made available under a CC-BY-NC-ND 4.0 International license .

589 such as blood serum, was possibly limited due to different platforms used by other studies. In 590 future studies we will extend our analysis to serum metabolomics data and seek replication of our

591 findings to facilitate further identification of novel biomarkers for AD.

592

593 Data availability

594 Metabolomics data from the Knight ADRC donors generated for this study are available at the 595 NIAGADS and can be accessed at https://www.niagads.org/knight-adrc-collection. Data 596 generated from the DIAN cohort can be requested at https://dian.wustl.edu/our-research/for597 investigators/diantu-investigator-resources/dian-tu-biospecimen-request-form/. We have 598 accessed data from the ADNI (https://adni.loni.usc.edu, accessed 18 December, 2020), and 599 ROSMAP (https://synapse.org/\#!Synapse:syn26007829, accessed 18 December, 2020 and 600 https://synapse.org/\#!Synapse:syn26007830, accessed 30 July, 2021). Additional phenotypic 601 data for the ROSMAP studies is available through the Rush AD Center Resource Sharing Hub 602 (https://www.radc.rush.edu).

603 
medRxiv preprint doi: https://doi.org/10.1101/2021.11.04.21265941; this version posted November 30, 2021. The copyright holder for this preprint (which was not certified by peer review) is the author/funder, who has granted medRxiv a license to display the preprint in perpetuity.

It is made available under a CC-BY-NC-ND 4.0 International license .

604 Funding

605 This work was possible thanks to the following governmental grants from the National institute of

606 Health: NIA R01AG057777, RO1AG057777-02S1, K99AG061281, P30AG066444, 607 P01AGO26276, NINDS R01NS118146 (BAB), R01AG044546 (CC), P01AG003991 (CC, JCM), 608 RF1AG053303 (CC), RF1AG058501 (CC), U01AG058922 (CC), and the Chan Zuckerberg 609 Initiative (CZI). O.H. is an Archer Foundation Research Scientist.

610 This work was supported by access to equipment made possible by the Hope Center for 611 Neurological Disorders, the NeuroGenomics and Informatics Center (NGl: 612 https://neurogenomics.wustl.edu/) and the Departments of Neurology and Psychiatry at 613 Washington University School of Medicine.

614 Acknowledgements

615 We thank contributors who collected samples used in this study and patients and their families, 616 whose help and participation made this work possible.

617 Dominantly Inherited Alzheimer Network (DIAN) resources

618 Data collection and sharing for this project was supported by The Dominantly Inherited Alzheimer 619 Network (DIAN, U19AG032438) funded by the National Institute on Aging (NIA), the Alzheimer's 620 Association (SG-20-690363-DIAN), the German Center for Neurodegenerative Diseases (DZNE), 621 Raul Carrea Institute for Neurological Research (FLENI), Partial support by the Research and 622 Development Grants for Dementia from Japan Agency for Medical Research and Development, 623 AMED, and the Korea Health Technology R\&D Project through the Korea Health Industry 624 Development Institute (KHIDI), Spanish Institute of Health Carlos III (ISCIII), Canadian Institutes 625 of Health Research (ClHR), Canadian Consortium of Neurodegeneration and Aging, Brain 626 Canada Foundation, and Fonds de Recherche du Québec - Santé. This manuscript has been 627 reviewed by DIAN Study investigators for scientific content and consistency of data interpretation 628 with previous DIAN Study publications. We acknowledge the altruism of the participants and their 629 families and contributions of the DIAN research and support staff at each of the participating sites 630 for their contributions to this study.

631 DIAN Study Group

632 Sarah Adams, Ricardo Allegri, Aki Araki, Nicolas Barthelemy, Randall Bateman, Jacob 633 Bechara,Tammie Benzinger, Sarah Berman, Courtney Bodge, Susan Brandon, William (Bill) 634 Brooks, Jared Brosch, Jill Buck, Virginia Buckles, Kathleen Carter, Lisa Cash, Charlie Chen, 
Jasmeer Chhatwal, Patricio Chrem, Jasmin Chua, Helena Chui, Carlos Cruchaga, Gregory S Day, Chrismary De La Cruz, Darcy Denner, Anna Diffenbacher, Aylin Dincer, Tamara Donahue, Jane Douglas, Duc Duong, Noelia Egido, Bianca Esposito, Anne Fagan, Marty Farlow, Becca Feldman, Colleen Fitzpatrick, Shaney Flores, Nick Fox, Erin Franklin, Nelly Friedrichsen, Hisako Fujii, Samantha Gardener, Bernardino Ghetti, Alison Goate, Sarah Goldberg, Jill Goldman, Alyssa Gonzalez, Brian Gordon, Susanne Gräber-Sultan, Neill Graff-Radford, Morgan Graham, Julia Gray, Emily Gremminger, Miguel Grilo, Alex Groves, Christian Haass, Lisa Häsler, Jason Hassenstab, Cortaiga Hellm, Elizabeth Herries, Laura Hoechst-Swisher, Anna Hofmann, David oltzman, Russ Hornbeck, Yakushev Igor, Ryoko Ihara, Takeshi Ikeuchi, Snezana Ikonomovic, Kenji Ishii, Clifford Jack, Gina Jerome, Erik Johnson, Mathias Jucker, Celeste Karch, Stephan Käser, Kensaku Kasuga, Sarah Keefe, William (Bill) Klunk, Robert Koeppe, Deb Koudelis, Elke Kuder-Buletta, Christoph Laske, Allan Levey, Johannes Levin, Yan Li, Oscar Lopez, Jacob Marsh, Rita Martinez, Ralph Martins, Neal Scott Mason, Colin Masters, Kwasi Mawuenyega, Austin McCullough, Eric McDade, Arlene Mejia, Estrella Morenas-Rodriguez, John Morris, James MountzMD, Cath Mummery, Neelesh Nadkarni, Akemi Nagamatsu, Katie Neimeyer, Yoshiki Niimi, James Noble, Joanne Norton, Brigitte Nuscher, Antoinette O'Connor, Ulricke Obermüller, Riddhi Patira, Richard Perrin, Lingyan Ping, Oliver Preische, Alan Renton, John Ringman, Stephen Salloway, Peter Schofield, Michio Senda, Nick Seyfried, Kristine Shady, Hiroyuki Shimada, Wendy Sigurdson, Jennifer Smith, Lori Smith, Beth Snitz, Hamid Sohrabi, Sochenda Stephens, Kevin Taddei, Sarah Thompson, Jonathan Vöglein, Peter Wang, Qing Wang, Elise Weamer, Chengjie Xiong, Jinbin Xu, Xiong Xu

Alzheimer's Disease Metabolomics Consortium (ADMC)

The results published here are in whole or in part based on data obtained from the AD Knowledge Portal (https://adknowledgeportal.org). Metabolomics data is provided by the Alzheimer's Disease Metabolomics Consortium (ADMC) and funded wholly or in part by the following grants and supplements thereto: NIA R01AG046171, RF1AG051550, 3U01AG024904-09S4, RF1AG057452, R01AG059093, RF1AG058942, U01AG061359, U19AG063744 and FNIH: \#DAOU16AMPA awarded to Dr. Kaddurah-Daouk at Duke University in partnership with a large number of academic institutions. As such, the investigators within the ADMC, not listed specifically in this publication's author's list, provided data along with its pre-processing and prepared it for analysis, but did not participate in analysis or writing of this manuscript. A complete listing of ADMC investigators can be found at: https://sites.duke.edu/adnimetab/team/. 
668

669

670

671

672

673

674

675

676

677

678

679

680

681

682

683

684

685

686

687

688

689

690

691

692

693

694

695

696

697

698

699

700

\section{ADNI}

Data collection and sharing for this project was funded by the Alzheimer's Disease Neuroimaging Initiative (ADNI) (National Institutes of Health Grant U01 AG024904) and DOD ADNI (Department of Defense award number W81XWH-12-2-0012). ADNI is funded by the National Institute on Aging, the National Institute of Biomedical Imaging and Bioengineering, and through generous contributions from the following: AbbVie, Alzheimer's Association; Alzheimer's Drug Discovery Foundation; Araclon Biotech; BioClinica, Inc.; Biogen; Bristol-Myers Squibb Company; CereSpir, Inc.; Cogstate; Eisai Inc.; Elan Pharmaceuticals, Inc.; Eli Lilly and Company; Eurolmmun; F. Hoffmann-La Roche Ltd and its affiliated company Genentech, Inc.; Fujirebio; GE Healthcare; IXICO Ltd.;Janssen Alzheimer Immunotherapy Research \& Development, LLC.; Johnson \& Johnson Pharmaceutical Research \& Development LLC.; Lumosity; Lundbeck; Merck \& Co., Inc.;Meso Scale Diagnostics, LLC.; NeuroRx Research; Neurotrack Technologies; Novartis Pharmaceuticals Corporation; Pfizer Inc.; Piramal Imaging; Servier; Takeda Pharmaceutical Company; and Transition Therapeutics. The Canadian Institutes of Health Research is providing funds to support ADNI clinical sites in Canada. Private sector contributions are facilitated by the Foundation for the National Institutes of Health (www.fnih.org). The grantee organization is the Northern California Institute for Research and Education, and the study is coordinated by the Alzheimer's Therapeutic Research Institute at the University of Southern California. ADNI data are disseminated by the Laboratory for Neuro Imaging at the University of Southern California.

\section{$\underline{\text { ROSMAP }}$}

Study data were provided by the Rush Alzheimer's Disease Center, Rush University Medical Center, Chicago. Data collection was supported through funding by NIA grants P30AG10161 (ROS), R01AG15819 (ROSMAP; genomics and RNAseq), R01AG17917 (MAP), R01AG30146, R01AG36042 (5hC methylation, ATACseq), RC2AG036547 (H3K9Ac), R01AG36836 (RNAseq), R01AG48015 (monocyte RNAseq) RF1AG57473 (single nucleus RNAseq), U01AG32984 (genomic and whole exome sequencing), U01AG46152 (ROSMAP AMP-AD, targeted proteomics), U01AG46161(TMT proteomics), U01AG61356 (whole genome sequencing, targeted proteomics, ROSMAP AMP-AD), the Illinois Department of Public Health (ROSMAP), and the Translational Genomics Research Institute (genomic). Additional phenotypic data can be requested at www.radc.rush.edu. Study data were provided through NIA grant 3R01AG04617102S2 awarded to Rima Kaddurah-Daouk at Duke University, based on specimens provided by the Rush Alzheimer's Disease Center, Rush University Medical Center, Chicago, where data collection was supported through funding by NIA grants P30AG10161, R01AG15819, 
medRxiv preprint doi: https://doi.org/10.1101/2021.11.04.21265941; this version posted November 30, 2021. The copyright holder for this preprint (which was not certified by peer review) is the author/funder, who has granted medRxiv a license to display the preprint in perpetuity.

It is made available under a CC-BY-NC-ND 4.0 International license .

701 R01AG17917, R01AG30146, R01AG36836, U01AG32984, U01AG46152, the Illinois Department

702 of Public Health, and the Translational Genomics Research Institute.

703 Additional Acknowledgements

704 We would like to pay our gratitude and respects to our friend and collaborator, Jorge Bahena.

705 Jorge was a remarkable scientist and respected colleague who earned his master's degree in

706 biostatistics from Washington University School of Medicine, and passed away in October 2021

707 as a doctoral student at Vanderbilt University. His valuable contributions to this and many other

708 endeavors will not be forgotten.

\section{Financial disclosures}

710 CC receives research support from: Biogen, EISAI, Alector and Parabon. The funders of the study

711 had no role in the collection, analysis, or interpretation of data; in the writing of the report; or in

712 the decision to submit the paper for publication. CC is a member of the advisory board of Vivid

713 genetics, Halia Therapeutics and ADx Healthcare. 
715

716

717

718

719

720

721

722

723

724

725

726

727

728

729

730

731

732

733

734

735

736

737

738

739

740

741

742

743

744

\section{References}

1. Zhao, N. et al. Alzheimer's risk factors age, APOE genotype, and sex drive distinct molecular pathways. Neuron 106, 727 (2020).

2. Badhwar, A. et al. A multiomics approach to heterogeneity in Alzheimer's disease: focused review and roadmap. Brain 143, 1315 (2020).

3. Holtzman, D. M., Morris, J. C. \& Goate, A. M. Alzheimer's disease: the challenge of the second century. Sci. Transl. Med. 3, (2011).

4. Pagani, M. et al. Early identification of MCl converting to AD: a FDG PET study. Eur. J. Nucl. Med. Mol. Imaging 44, 2042-2052 (2017).

5. González-Domínguez, R., Sayago, A. \& Fernández-Recamales, Á. Metabolomics in Alzheimer's disease: The need of complementary analytical platforms for the identification of biomarkers to unravel the underlying pathology. J. Chromatogr. B 1071, 75-92 (2017).

6. Yiannopoulou, K. G. \& Papageorgiou, S. G. Current and Future Treatments in Alzheimer Disease: An Update. J. Cent. Nerv. Syst. Dis. 12, 117957352090739 (2020).

7. Ferrucci, L. The Baltimore Longitudinal Study of Aging (BLSA): a 50-year-long journey and plans for the future. J. Gerontol. A. Biol. Sci. Med. Sci. 63, 1416-1419 (2008).

8. Mueller, S. G. et al. The Alzheimer's disease neuroimaging initiative. Neuroimaging Clin. N. Am. 15, 869-877 (2005).

9. Shock, N. W. et al. Normal Human Aging: The Baltimore Longitudinal Study on Aging. (1984).

10. Varma, V. R. et al. Brain and blood metabolite signatures of pathology and progression in Alzheimer disease: A targeted metabolomics study. PLOS Med. 15, e1002482 (2018).

11. Stamate, D. et al. A metabolite-based machine learning approach to diagnose Alzheimertype dementia in blood: Results from the European Medical Information Framework for Alzheimer disease biomarker discovery cohort. Alzheimer's Dement. Transl. Res. Clin. Interv. 5, 933-938 (2019).

12. Karch, C. M. \& Goate, A. M. Alzheimer's disease risk genes and mechanisms of disease pathogenesis. (2014) doi:10.1016/j.biopsych.2014.05.006.

13. Jansen, I. E. et al. Genome-wide meta-analysis identifies new loci and functional 
medRxiv preprint doi: https://doi.org/10.1101/2021.11.04.21265941; this version posted November 30, 2021. The copyright holder for this preprint (which was not certified by peer review) is the author/funder, who has granted medRxiv a license to display the preprint in perpetuity.

It is made available under a CC-BY-NC-ND 4.0 International license .

745

746

747

748

749

750

751

752

753

754

755

756

757

758

759

760

761

762

763

764

765

766

767

768

769

770

771

772

773

774

pathways influencing Alzheimer's disease risk. Nat. Genet. 2019513 51, 404-413 (2019).

14. Tan, M.-S. et al. Associations of Alzheimer's disease risk variants with gene expression, amyloidosis, tauopathy, and neurodegeneration. Alzheimer's Res. Ther. 2021131 13, 1$11(2021)$.

15. Haass, C., Kaether, C., Thinakaran, G. \& Sisodia, S. Trafficking and Proteolytic Processing of APP. Cold Spring Harb. Perspect. Med. 2, (2012).

16. Zhao, Y. et al. TREM2 Is a Receptor for $\beta$-Amyloid that Mediates Microglial Function. Neuron 97, 1023-1031.e7 (2018).

17. Zhong, L. et al. Amyloid-beta modulates microglial responses by binding to the triggering receptor expressed on myeloid cells 2 (TREM2). Mol. Neurodegener. 13, (2018).

18. Ulland, T. K. \& Colonna, M. TREM2 - a key player in microglial biology and Alzheimer disease. Nat. Rev. Neurol. 20181411 14, 667-675 (2018).

19. Arnold, M. et al. Sex and APOE \&4 genotype modify the Alzheimer's disease serum metabolome. Nat. Commun. 11, (2020).

20. Morris, J. C. The clinical dementia rating (cdr): Current version and scoring rules. Neurology 43, 2412-2414 (1993).

21. Coats, M. \& Morris, J. C. Antecedent biomarkers of Alzheimer's disease: the adult children study. J. Geriatr. Psychiatry Neurol. 18, 242-4 (2005).

22. Bateman, R. J. et al. Clinical and Biomarker Changes in Dominantly Inherited Alzheimer's Disease. N. Engl. J. Med. 367, 795 (2012).

23. Berg, L. et al. Clinicopathologic Studies in Cognitively Healthy Aging and Alzheimer Disease: Relation of Histologic Markers to Dementia Severity, Age, Sex, and Apolipoprotein E Genotype. Arch. Neurol. 55, 326-335 (1998).

24. Yang, C. et al. Genomic atlas of the proteome from brain, CSF and plasma prioritizes proteins implicated in neurological disorders. Nat. Neurosci. 24, 1302-1312 (2021).

25. Dube, U. et al. An atlas of cortical circular RNA expression in Alzheimer disease brains demonstrates clinical and pathological associations. Nat. Neurosci. 22, 1903 (2019).

26. Del-Aguila, J. L. et al. A single-nuclei RNA sequencing study of Mendelian and sporadic AD in the human brain. Alzheimers. Res. Ther. 11, (2019). 
medRxiv preprint doi: https://doi.org/10.1101/2021.11.04.21265941; this version posted November 30, 2021. The copyright holder for this preprint (which was not certified by peer review) is the author/funder, who has granted medRxiv a license to display the preprint in perpetuity.

It is made available under a CC-BY-NC-ND 4.0 International license .

775

776

777

778

779

780

781

782

783

784

785

786

787

788

789

790

791

792

793

794

795

796

797

798

799

800

801

802

803

804

27. Del-Aguila, J. L. et al. TREM2 brain transcript-specific studies in AD and TREM2 mutation carriers. Mol. Neurodegener. 14, (2019).

28. Jiang, S. et al. Integrative system biology analyses of CRISPR-edited iPSC-derived neurons and human brains reveal deficiencies of presynaptic signaling in FTLD and PSP. Transl. Psychiatry 8, 265 (2018).

29. Li, Z. et al. Genetic variants associated with Alzheimer's disease confer different cerebral cortex cell-type population structure. Genome Med. 10, (2018).

30. Crary, J. F. et al. Primary age-related tauopathy (PART): a common pathology associated with human aging. Acta Neuropathol. 128, 755 (2014).

31. Hickman, R. A., Flowers, X. E. \& Wisniewski, T. Primary Age-Related Tauopathy (PART): Addressing the Spectrum of Neuronal Tauopathic Changes in the Aging Brain. Curr. Neurol. Neurosci. Rep. 20, 39 (2020).

32. Toledo, J. B. et al. Metabolic network failures in Alzheimer's disease: A biochemical road map. Alzheimer's Dement. 13, 965-984 (2017).

33. St John-Williams, L. et al. Targeted metabolomics and medication classification data from participants in the ADNI1 cohort. Sci. Data 4, (2017).

34. Batra, R. et al. The landscape of metabolic brain alterations in Alzheimer's disease. bioRxiv 2021.11.15.468698 (2021) doi:10.1101/2021.11.15.468698.

35. Bennett, D. A. et al. Neuropathology of older persons without cognitive impairment from two community-based studies. Neurology 66, 1837-1844 (2006).

36. Bennett, D. A., Schneider, J. A., Arvanitakis, Z. \& Wilson, R. S. OVERVIEW AND FINDINGS FROM THE RELIGIOUS ORDERS STUDY. Curr. Alzheimer Res. 9, 628 (2012).

37. JA, S., Z, A., W, B. \& DA, B. Mixed brain pathologies account for most dementia cases in community-dwelling older persons. Neurology 69, 2197-2204 (2007).

38. Team, the A. ADNIMERGE: Alzheimer's Disease Neuroimaging Initiative. R package version 0.0.1 (2018).

39. Saykin, A. J. et al. Alzheimer's Disease Neuroimaging Initiative biomarkers as quantitative phenotypes: Genetics core aims, progress, and plans. Alzheimer's Dement. 6, 265-273 (2010). 
medRxiv preprint doi: https://doi.org/10.1101/2021.11.04.21265941; this version posted November 30, 2021. The copyright holder for this preprint (which was not certified by peer review) is the author/funder, who has granted medRxiv a license to display the preprint in perpetuity.

It is made available under a CC-BY-NC-ND 4.0 International license .

805

806

807

808

809

810

811

812

813

814

815

816

817

818

819

820

821

822

823

824

825

826

827

828

829

830

831

832

833

834

40. Lê, S., Josse, J. \& Husson, F. FactoMineR: An R package for multivariate analysis. J. Stat. Softw. 25, 1-18 (2008).

41. R Core Team. R: A language and environment for statistical computing. (2020).

42. Langfelder, P. \& Horvath, S. WGCNA: An R package for weighted correlation network analysis. BMC Bioinformatics 9, 1-13 (2008).

43. Gu, Z., Eils, R. \& Schlesner, M. Complex heatmaps reveal patterns and correlations in multidimensional genomic data. Bioinformatics 32, 2847-2849 (2016).

44. Gu, Z., Gu, L., Eils, R., Schlesner, M. \& Brors, B. Circlize implements and enhances circular visualization in R. Bioinformatics 30, 2811-2812 (2014).

45. Xia, J., Wishart, D. S. \& Valencia, A. MetPA: A web-based metabolomics tool for pathway analysis and visualization. in Bioinformatics vol. 27 2342-2344 (Oxford University Press, 2011).

46. Chong, J. et al. MetaboAnalyst 4.0: Towards more transparent and integrative metabolomics analysis. Nucleic Acids Res. 46, W486-W494 (2018).

47. Kamburov, A., Cavill, R., Ebbels, T. M. D., Herwig, R. \& Keun, H. C. Integrated pathwaylevel analysis of transcriptomics and metabolomics data with IMPaLA. Bioinformatics 27, 2917-2918 (2011).

48. Kanehisa, M. \& Goto, S. KEGG: Kyoto Encyclopedia of Genes and Genomes. Nucleic Acids Research vol. 28 27-30 (2000).

49. Kanehisa, M., Sato, Y., Furumichi, M., Morishima, K. \& Tanabe, M. New approach for understanding genome variations in KEGG. Nucleic Acids Res. 47, D590-D595 (2019).

50. Kanehisa, M., Furumichi, M., Sato, Y., Ishiguro-Watanabe, M. \& Tanabe, M. KEGG: Integrating viruses and cellular organisms. Nucleic Acids Res. 49, D545-D551 (2021).

51. Chang, W., Cheng, J., Allaire, J., Xie, Y. \& McPherson, J. shiny: Web Application Framework for R. (2019).

52. Chang, W. \& Borges Ribeiro, B. shinydashboard: Create Dashboards with 'Shiny'. (2018).

53. Granjon, D. shinydashboardPlus: Add More 'AdminLTE2' Components to shinydashboard. (2020).

54. Sievert, C. Interactive Web-Based Data Visualization with R, plotly, and shiny. (Chapman and Hall/CRC, 2020). 
medRxiv preprint doi: https://doi.org/10.1101/2021.11.04.21265941; this version posted November 30, 2021. The copyright holder for this preprint (which was not certified by peer review) is the author/funder, who has granted medRxiv a license to display the preprint in perpetuity.

It is made available under a CC-BY-NC-ND 4.0 International license .

835

836

837

838

839

840

841

842

843

844

845

846

847

848

849

850

851

852

853

854

855

856

857

858

859

860

861

862

863

55. Xie, Y., Cheng, J. \& Tan, X. DT: A Wrapper of the JavaScript Library ‘DataTables'. (2020).

56. Attali, D. shinyjs: Easily Improve the User Experience of Your Shiny Apps in Seconds. (2018).

57. Vaidyanathan, R. \& Xie, Y. htmlwidgets: HTML Widgets for R. (2019).

58. Neuwirth, E. RColorBrewer: ColorBrewer Palettes. (2014).

59. Zhu, H. kableExtra: Construct Complex Table with 'kable' and Pipe Syntax. (2019).

60. Wickham, H., Francois, R., Henry, L. \& Muller, K. dplyr: A Grammar of Data Manipulation. (2020).

61. González-Domínguez, R., García-Barrera, T. \& Gómez-Ariza, J. L. Metabolite profiling for the identification of altered metabolic pathways in Alzheimer's disease. J. Pharm.

Biomed. Anal. 107, 75-81 (2015).

62. Wang, R. \& Reddy, P. H. Role of Glutamate and NMDA Receptors in Alzheimer's Disease. Journal of Alzheimer's Disease vol. 57 1041-1048 (2017).

63. Liu, H., Harrell, L. E., Shenvi, S., Hagen, T. \& Liu, R. M. Gender differences in glutathione metabolism in Alzheimer's disease. J. Neurosci. Res. 79, 861-867 (2005).

64. Snowden, S. G. et al. Neurotransmitter Imbalance in the Brain and Alzheimer's Disease Pathology. J. Alzheimer's Dis. 72, 35-43 (2019).

65. Zhao, J. et al. A comprehensive metabolomics investigation of hippocampus, serum, and feces affected by chronic fluoxetine treatment using the chronic unpredictable mild stress mouse model of depression. Sci. Rep. 9, 1-11 (2019).

66. Huo, Z. et al. Brain and blood metabolome for Alzheimer's dementia: findings from a targeted metabolomics analysis. Neurobiol. Aging 86, 123-133 (2020).

67. Kim, S. et al. PubChem 2019 update: Improved access to chemical data. Nucleic Acids Res. 47, D1102-D1109 (2019).

68. Wishart, D. S. et al. HMDB: The human metabolome database. Nucleic Acids Res. 35, (2007).

69. Gordon, B. A. et al. Tau PET in autosomal dominant Alzheimer's disease: relationship with cognition, dementia and other biomarkers. Brain 142, 1063-1076 (2019). 
medRxiv preprint doi: https://doi.org/10.1101/2021.11.04.21265941; this version posted November 30, 2021. The copyright holder for this preprint (which was not certified by peer review) is the author/funder, who has granted medRxiv a license to display the preprint in perpetuity.

It is made available under a CC-BY-NC-ND 4.0 International license .

864

865

866

867

868

869

870

871

872

873

874

875

876

877

878

879

880

881

882

883

884

885

886

887

888

889

890

891

892

893

70. Peña-Bautista, C. et al. Plasma alterations in cholinergic and serotonergic systems in early Alzheimer Disease: Diagnosis utility. Clin. Chim. Acta 500, 233-240 (2020).

71. Graham, S. F. et al. Untargeted metabolomic analysis of human plasma indicates differentially affected polyamine and L-Arginine metabolism in mild cognitive impairment subjects converting to alzheimer's disease. PLoS One 10, (2015).

72. Mahajan, U. V. et al. Dysregulation of multiple metabolic networks related to brain transmethylation and polyamine pathways in Alzheimer disease: A targeted metabolomic and transcriptomic study. PLoS Med. 17, e1003012 (2020).

73. Hamada-Kanazawa, M. et al. $\beta$-citryl-L-glutamate acts as an iron carrier to activate aconitase activity. Biol. Pharm. Bull. 34, 1455-1464 (2011).

74. Lewis, E. D., Meydani, S. N. \& Wu, D. Regulatory role of vitamin E in the immune system and inflammation. IUBMB Life 71, 487-494 (2019).

75. Freedman, J. E., Farhat, J. H., Loscalzo, J. \& Keaney Jr., J. F. alpha-tocopherol inhibits aggregation of human platelets by a protein kinase C-dependent mechanism. Circulation 94, 2434-2440 (1996).

76. Freedman, J. E. \& Keaney Jr., J. F. Vitamin E Inhibition of Platelet Aggregation Is Independent of Antioxidant Activity. J. Nutr. 131, 374S-377S (2001).

77. Akanmu, D., Cecchini, R., Aruoma, O. I. \& Halliwell, B. The antioxidant action of ergothioneine. Arch. Biochem. Biophys. 288, 10-16 (1991).

78. Halliwell, B., Cheah, I. K. \& Tang, R. M. Y. Ergothioneine - a diet-derived antioxidant with therapeutic potential. FEBS Lett. 592, 3357-3366 (2018).

79. Tang, R. M. Y., Cheah, I. K.-M., Yew, T. S. K. \& Halliwell, B. Distribution and accumulation of dietary ergothioneine and its metabolites in mouse tissues. Sci. Reports $2018818,1-15$ (2018).

80. Cheah, I. K., Feng, L., Tang, R. M. Y., Lim, K. H. C. \& Halliwell, B. Ergothioneine levels in an elderly population decrease with age and incidence of cognitive decline; a risk factor for neurodegeneration? Biochem. Biophys. Res. Commun. 478, 162-167 (2016).

81. Yang, N. C. et al. Ergothioneine protects against neuronal injury induced by $\beta$-amyloid in mice. Food Chem. Toxicol. 50, 3902-3911 (2012).

82. Cirrito, J. R. et al. Serotonin signaling is associated with lower amyloid- $\beta$ levels and 
medRxiv preprint doi: https://doi.org/10.1101/2021.11.04.21265941; this version posted November 30, 2021. The copyright holder for this preprint (which was not certified by peer review) is the author/funder, who has granted medRxiv a license to display the preprint in perpetuity. It is made available under a CC-BY-NC-ND 4.0 International license .

894

895

896

897

898

899

900

901

902

903

plaques in transgenic mice and humans. Proc. Natl. Acad. Sci. 108, 14968-14973 (2011).

83. Cirrito, J. R. et al. Effect of escitalopram on $A \beta$ levels and plaque load in an Alzheimer mouse model. Neurology 95, e2666-e2674 (2020).

84. Sheline, Y. I. et al. Effect of escitalopram dose and treatment duration on CSF A $\beta$ levels in healthy older adults. Neurology 95, e2658-e2665 (2020).

85. Schroeder, S. et al. Dietary spermidine improves cognitive function. Cell Rep. 35, (2021).

86. Liang, Y. T. et al. elF5A hypusination, boosted by dietary spermidine, protects from premature brain aging and mitochondrial dysfunction. Cell Rep. 35, (2021). 
Table 1. Summary statistics for the six datasets included in this study

\begin{tabular}{|c|c|c|c|c|c|c|c|c|c|c|c|c|c|}
\hline & & \multirow[b]{2}{*}{ Condition } & \multirow[b]{2}{*}{$\mathrm{N}$} & \multirow[b]{2}{*}{$\mathrm{Age}^{*}$} & \multirow[b]{2}{*}{$\% \mathrm{Fe}$} & \multicolumn{4}{|c|}{ Braak (Tau) } & \multicolumn{4}{|c|}{ CDR } \\
\hline & & & & & & \%APOE4+ & $0-1 I I$ & IV-VI & Not avail. & $<=0.5$ & $1-2$ & 3 & PMI \\
\hline \multirow{5}{*}{ Discovery } & \multirow{5}{*}{$\begin{array}{c}\text { WUSM } \\
\text { (parietal brain) }\end{array}$} & ADAD & 25 & $54 \pm 13.9$ & $32 \%$ & $36 \%$ & 0 & 20 & 5 & 0 & 0 & 25 & 13.3 \\
\hline & & sAD & 305 & $84 \pm 8.8$ & $62 \%$ & $56 \%$ & 23 & 195 & 87 & 0 & 23 & 282 & 12.5 \\
\hline & & TREM2 & 21 & $84 \pm 7.5$ & $52 \%$ & $57 \%$ & 3 & 12 & 6 & 0 & 1 & 20 & 14.1 \\
\hline & & Presymptomatic & 15 & $87 \pm 9.7$ & $60 \%$ & $53 \%$ & 3 & 8 & 4 & 15 & 0 & 0 & 13.6 \\
\hline & & Control & 26 & $88 \pm 9.1$ & $69 \%$ & $12 \%$ & 20 & 1 & 5 & 26 & 0 & 0 & 10.0 \\
\hline \multirow{10}{*}{ Replication } & \multirow{2}{*}{$\begin{array}{l}\text { ROSMAP (DLPFC } \\
\text { brain Metabolon) }\end{array}$} & $s A D$ & 233 & $89 \pm 2.9$ & $73 \%$ & $38 \%$ & 15 & 218 & 0 & & & & 8.1 \\
\hline & & Control & 94 & $86 \pm 4.7$ & $59 \%$ & $10 \%$ & 94 & 0 & 0 & & & & 7.7 \\
\hline & \multirow{2}{*}{$\begin{array}{l}\text { ROSMAP (DLPFC } \\
\text { brain p180) }\end{array}$} & sAD & 43 & $88 \pm 2.8$ & $19 \%$ & $29 \%$ & 3 & 40 & 0 & & & & 9.6 \\
\hline & & Control & 23 & $87 \pm 3.8$ & $35 \%$ & $7 \%$ & 23 & 0 & 0 & & & & 8.7 \\
\hline & \multirow{2}{*}{ ROSMAP (serum) } & SAD & 36 & $87 \pm 3.8$ & $81 \%$ & $8 \%$ & & & & & & & \\
\hline & & Control & 55 & $87 \pm 4.4$ & $69 \%$ & $0 \%$ & & & & & & & \\
\hline & \multirow{2}{*}{ ADNI1 (serum) } & SAD & 184 & $75 \pm 7.5$ & $48 \%$ & $66 \%$ & & & & & & & \\
\hline & & Control & 224 & $76 \pm 5.0$ & $48 \%$ & $27 \%$ & & & & & & & \\
\hline & \multirow{2}{*}{ ADNI2GO (serum) } & SAD & 137 & $74 \pm 8.2$ & $42 \%$ & $66 \%$ & & & & & & & \\
\hline & & Control & 181 & $73 \pm 6.4$ & $51 \%$ & $28 \%$ & & & & & & & \\
\hline
\end{tabular}

$\mathrm{sAD}=$ Sporadic Alzheimer Disease; $\mathrm{CDR}=$ Clinical Dementia Rating; $\mathrm{PMI}=$ post mortem interval

${ }^{*}$ Age is age at death for brain samples, and age at baseline for serum samples. 
Table 2. Effects, $p$-values, and q-values for 16 metabolites which were significant after FDR correction in the ADAD vs CO comparisons and at least nominally significant in the AD vs CO and TREM2 vs CO comparisons in the WUSM cohort. Highlighted in bold are metabolites with significant $Q$ values in the AD vs CO or TREM2 vs CO comparisons.

\begin{tabular}{|c|c|c|c|c|c|c|c|c|c|c|c|c|}
\hline \multirow[b]{2}{*}{ Metabolite } & \multicolumn{3}{|c|}{ ADAD vs CO } & \multicolumn{3}{|c|}{ sAD vs CO } & \multicolumn{3}{|c|}{ TREM2 vs CO } & \multicolumn{3}{|c|}{$\begin{array}{c}\text { ROSMAP (DLPFC } \\
\text { Metabolon) }\end{array}$} \\
\hline & Effect & $p$-value & $q$-value & Effect & $p$-value & $q$-value & Effect & $p$-value & $q$-value & Effect & $p$-value & $q$-value \\
\hline aspartate & 0.160 & $1.1 \times 10^{-3}$ & $1.1 \times 10^{-2}$ & 0.085 & $2.2 \times 10^{-3}$ & $9.8 \times 10^{-2}$ & 0.123 & $6.0 \times 10^{-3}$ & 0.27 & 0.042 & $1.1 \times 10^{-2}$ & $5.4 \times 10^{-2}$ \\
\hline v-glutamylthreonine & 0.263 & $3.2 \times 10^{-3}$ & $2.3 \times 10^{-2}$ & 0.171 & $5.4 \times 10^{-4}$ & $5.5 \times 10^{-2}$ & 0.278 & $7.1 \times 10^{-4}$ & 0.11 & 0.095 & $5.1 \times 10^{-4}$ & $5.4 \times 10^{-3}$ \\
\hline$\beta$-citrylglutamate & -0.217 & $4.9 \times 10^{-5}$ & $2.2 \times 10^{-3}$ & -0.148 & $2.1 \times 10^{-4}$ & $3.6 \times 10^{-2}$ & -0.293 & $7.8 \times 10^{-7}$ & $4.9 \times 10^{-4}$ & -0.062 & $3.7 \times 10^{-4}$ & $4.2 \times 10^{-3}$ \\
\hline glutamate & 0.086 & $7.1 \times 10^{-3}$ & $3.9 \times 10^{-2}$ & 0.048 & $1.7 \times 10^{-2}$ & 0.36 & 0.071 & $6.5 \times 10^{-3}$ & 0.27 & 0.060 & $1.1 \times 10^{-8}$ & $1.4 \times 10^{-6}$ \\
\hline $\mathrm{N}$-acetylglutamate & -0.207 & $1.7 \times 10^{-5}$ & $1.0 \times 10^{-3}$ & -0.080 & $2.7 \times 10^{-3}$ & $9.8 \times 10^{-2}$ & -0.122 & $3.2 \times 10^{-3}$ & 0.19 & -0.033 & $6.8 \times 10^{-4}$ & $6.7 \times 10^{-3}$ \\
\hline ergothioneine & -0.255 & $8.3 \times 10^{-3}$ & $4.3 \times 10^{-2}$ & -0.213 & $8.0 \times 10^{-5}$ & $2.5 \times 10^{-2}$ & -0.274 & $7.5 \times 10^{-3}$ & 0.27 & -0.092 & $2.5 \times 10^{-2}$ & 0.10 \\
\hline 3-hydroxy-2-ethylpropionate & 0.177 & $2.0 \times 10^{-3}$ & $1.7 \times 10^{-2}$ & 0.099 & $4.4 \times 10^{-2}$ & 0.49 & 0.195 & $8.7 \times 10^{-3}$ & 0.27 & & & \\
\hline 1,5-anhydroglucitol (1,5-AG) & 0.310 & $6.6 \times 10^{-6}$ & $7.3 \times 10^{-4}$ & 0.131 & $1.1 \times 10^{-2}$ & 0.27 & 0.155 & $4.3 \times 10^{-2}$ & 0.47 & 0.088 & $4.4 \times 10^{-3}$ & $2.8 \times 10^{-2}$ \\
\hline 2-methylcitrate/homocitrate & -0.171 & $1.3 \times 10^{-3}$ & $1.2 \times 10^{-2}$ & -0.087 & $1.3 \times 10^{-2}$ & 0.30 & -0.118 & $2.9 \times 10^{-2}$ & 0.41 & -0.048 & $1.1 \times 10^{-2}$ & $5.4 \times 10^{-2}$ \\
\hline glutarate (C5-DC) & 0.252 & $7.3 \times 10^{-5}$ & $2.3 \times 10^{-3}$ & 0.121 & $5.7 \times 10^{-3}$ & 0.17 & 0.170 & $1.6 \times 10^{-2}$ & 0.35 & 0.090 & $1.5 \times 10^{-5}$ & $4.0 \times 10^{-4}$ \\
\hline CDP-choline & -0.095 & $9.6 \times 10^{-4}$ & $1.0 \times 10^{-2}$ & -0.040 & $4.6 \times 10^{-2}$ & 0.49 & -0.093 & $1.6 \times 10^{-3}$ & 0.14 & 0.042 & $7.5 \times 10^{-3}$ & $4.1 \times 10^{-2}$ \\
\hline CDP-ethanolamine & -0.102 & $6.1 \times 10^{-4}$ & $7.7 \times 10^{-3}$ & -0.054 & $2.8 \times 10^{-3}$ & $9.9 \times 10^{-2}$ & -0.128 & $1.8 \times 10^{-4}$ & $3.7 \times 10^{-2}$ & -0.024 & $8.2 \times 10^{-2}$ & 0.23 \\
\hline glycerophosphoinositol & -0.164 & $3.4 \times 10^{-4}$ & $5.3 \times 10^{-3}$ & -0.095 & $1.3 \times 10^{-3}$ & $8.0 \times 10^{-2}$ & -0.098 & $2.7 \times 10^{-2}$ & 0.41 & -0.021 & $4.5 \times 10^{-2}$ & 0.15 \\
\hline nicotinamide (vitamin B3) & -0.066 & $6.8 \times 10^{-3}$ & $3.7 \times 10^{-2}$ & -0.032 & $1.9 \times 10^{-2}$ & 0.36 & -0.056 & $9.4 \times 10^{-3}$ & 0.27 & 0.008 & 0.33 & 0.56 \\
\hline a-tocopherol (vitamin E) & -0.120 & $4.1 \times 10^{-5}$ & $2.0 \times 10^{-3}$ & -0.065 & $1.5 \times 10^{-3}$ & $8.0 \times 10^{-2}$ & -0.115 & $1.8 \times 10^{-5}$ & $5.6 \times 10^{-3}$ & -0.123 & $3.0 \times 10^{-5}$ & $5.7 \times 10^{-4}$ \\
\hline retinol (Vitamin A) & -0.287 & $1.3 \times 10^{-5}$ & $9.2 \times 10^{-4}$ & -0.156 & $8.0 \times 10^{-4}$ & $5.6 \times 10^{-2}$ & -0.192 & $1.4 \times 10^{-3}$ & 0.14 & -0.196 & $2.9 \mathrm{E}-04$ & $3.7 \times 10^{-3}$ \\
\hline
\end{tabular}


medRxiv preprint doi: https://doi.org/10.1101/2021.11.04.21265941; this version posted November 30, 2021. The copyright holder for this preprint (which was not certified by peer review) is the author/funder, who has granted medRxiv a license to display the preprint in perpetuity.

It is made available under a CC-BY-NC-ND 4.0 International license .

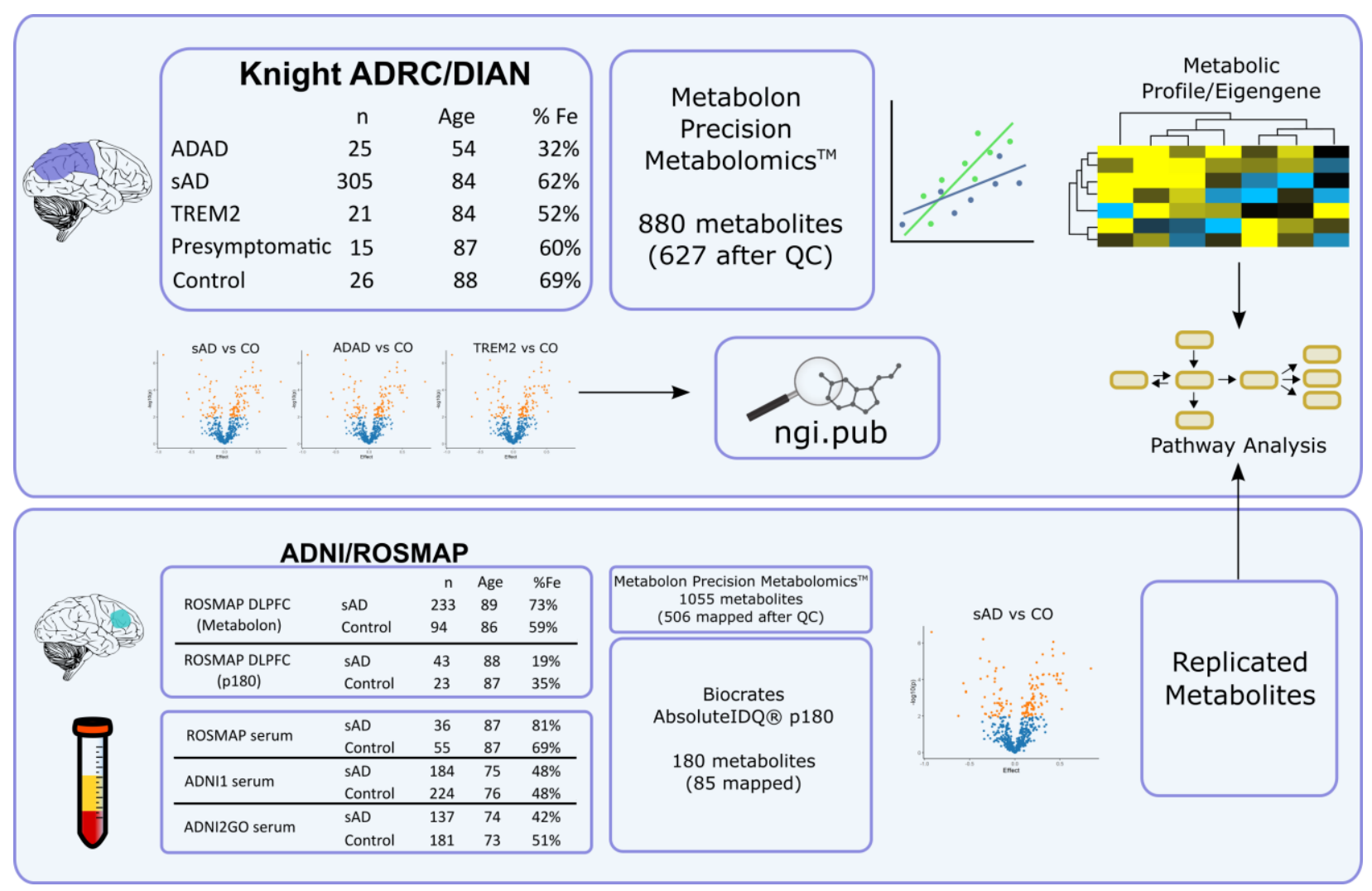

Figure 1. Study design. Parietal cortical tissue from donors to the Knight ADRC and DIAN were analyzed on the Metabolon Precision Metabolomics platform: autosomal dominant AD (ADAD, $n=25)$, sporadic $A D(s A D, n=305)$, TREM2 ( $n=21)$, Presymptomatic $(n=15)$, and healthy control $(\mathrm{CO}, \mathrm{n}=26)$. After quality control, 627 metabolites were tested for differential abundance via linear modeling. A metabolic profile was generated from 16 metabolites in common between groups. Pathway analysis was performed on the differentially abundant metabolites, and a web browser was created to share the data and results. Findings were validated in five independent datasets: dorsolateral prefrontal cortex (DLPFC) tissue from the ROSMAP cohort analyzed on the Metabolon platform ( $S A D n=233, C O n=94$ ), as well as four datasets quantified using the Biocrates p180 platform: DLPFC from the ROSMAP cohort ( $S A D=43, C O=23$ ), serum from the ROSMAP cohort ( $S A D n=36, C O n=55$ ), serum from the $A D N I 1$ cohort ( $S A D n=184, C O n=224$ ), and serum from the $A D N I G O / 2$ cohort ( $S A D n=137, C O n=181$ ). The ROSMAP Metabolon dataset was found to have 506 metabolites in common with the Knight ADRC/DIAN cohort after quality control. The p180 platform was found to have 85 metabolites in common with the Metabolon platform. 
medRxiv preprint doi: https://doi.org/10.1101/2021.11.04.21265941; this version posted November 30, 2021. The copyright holder for this preprint (which was not certified by peer review) is the author/funder, who has granted medRxiv a license to display the preprint in perpetuity.

It is made available under a CC-BY-NC-ND 4.0 International license .

A)

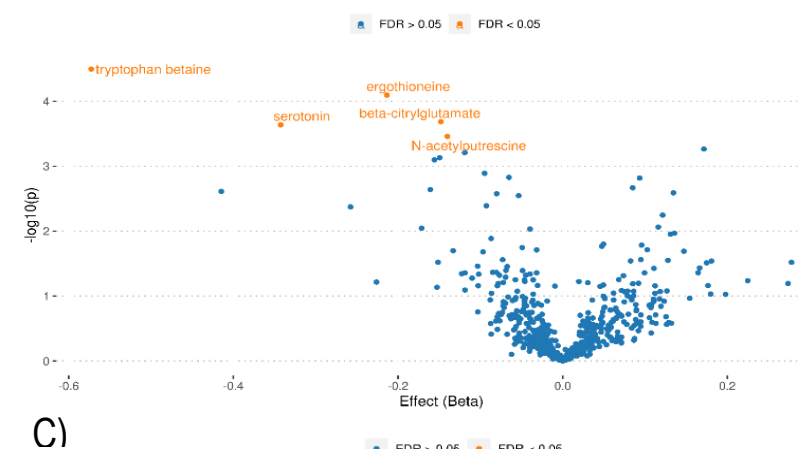

C)

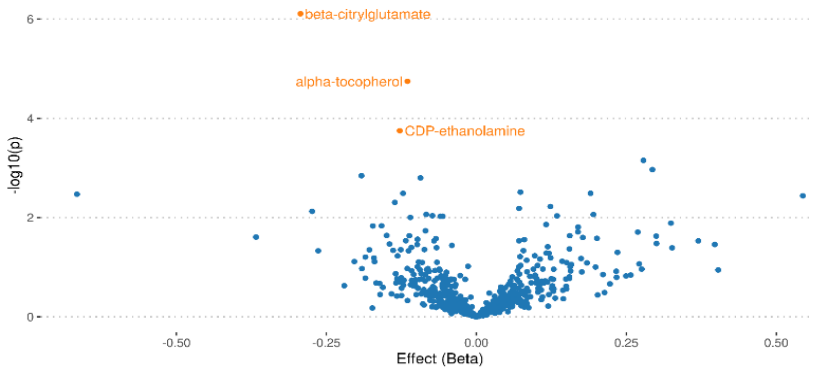

E)

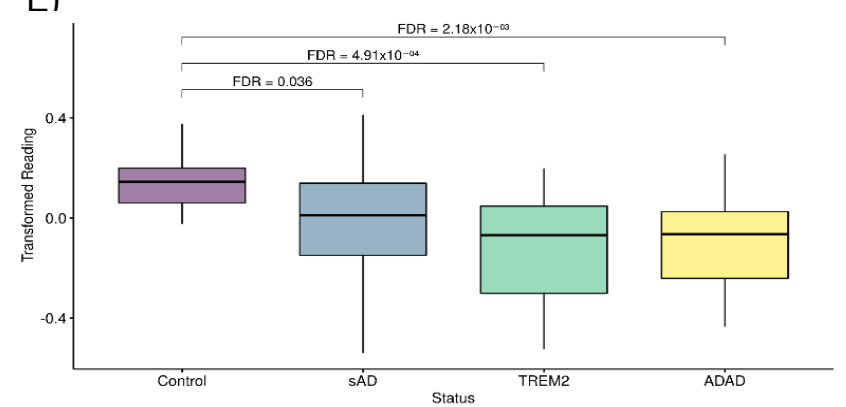

G)

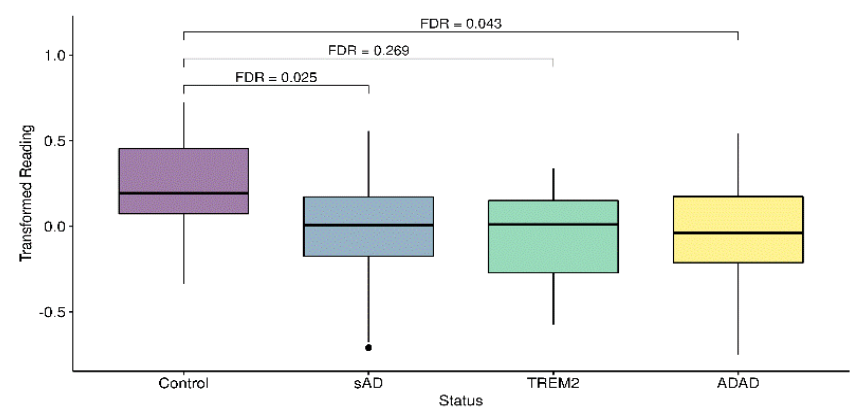

B)

D)
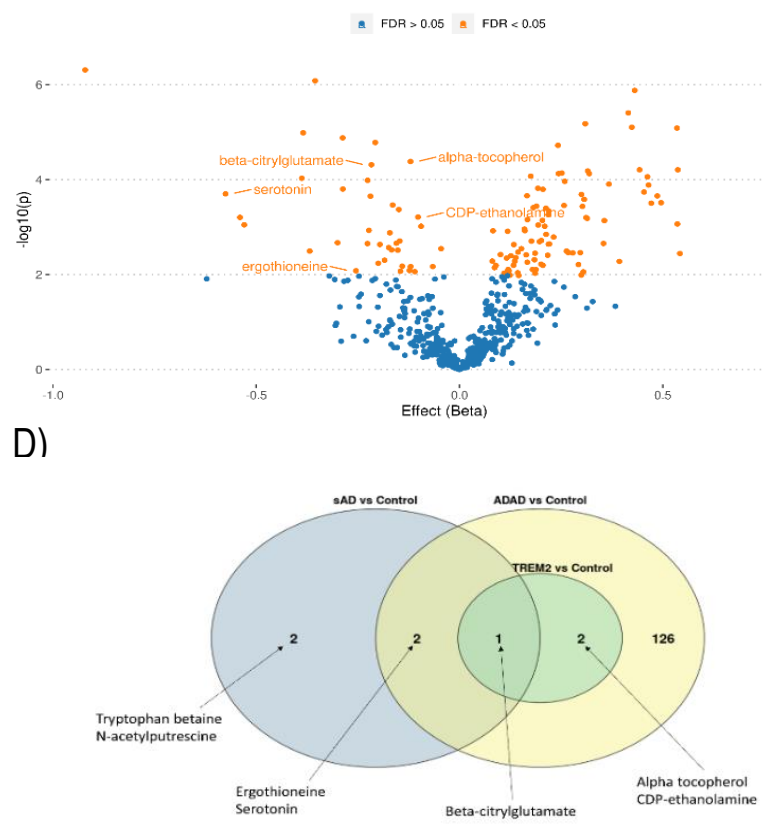

F)

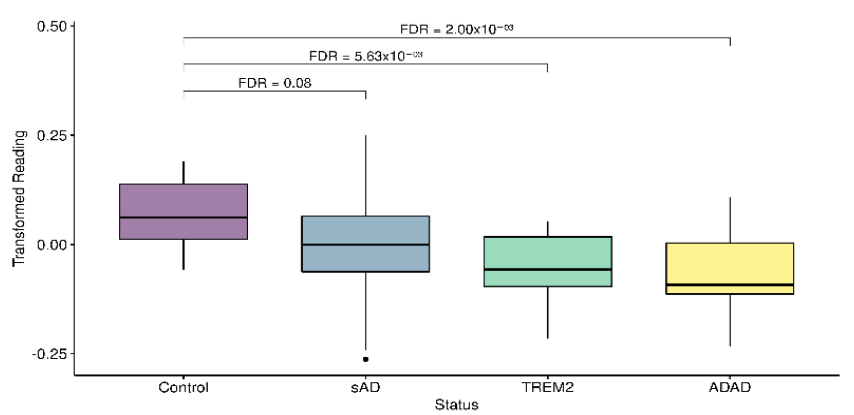

H)

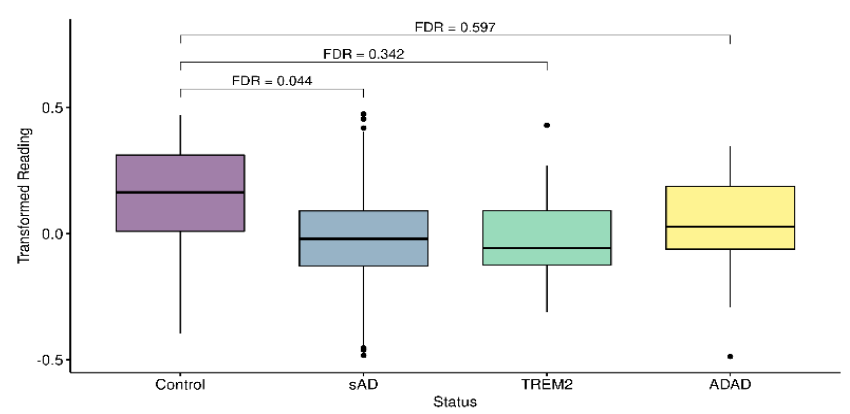

Figure 2. Association analysis in WUSM dataset. Volcano plots for $A$ ) $S A D$ vs $C O, B$ ) ADAD vs CO, C) TREM2 vs CO. D) Venn diagram. Box plots for abundance of top metabolites E) $\beta$ citrylglutamate. F) a-tocopherol, G) ergothioneine, H) $\mathrm{N}$-acetylputrescine. 


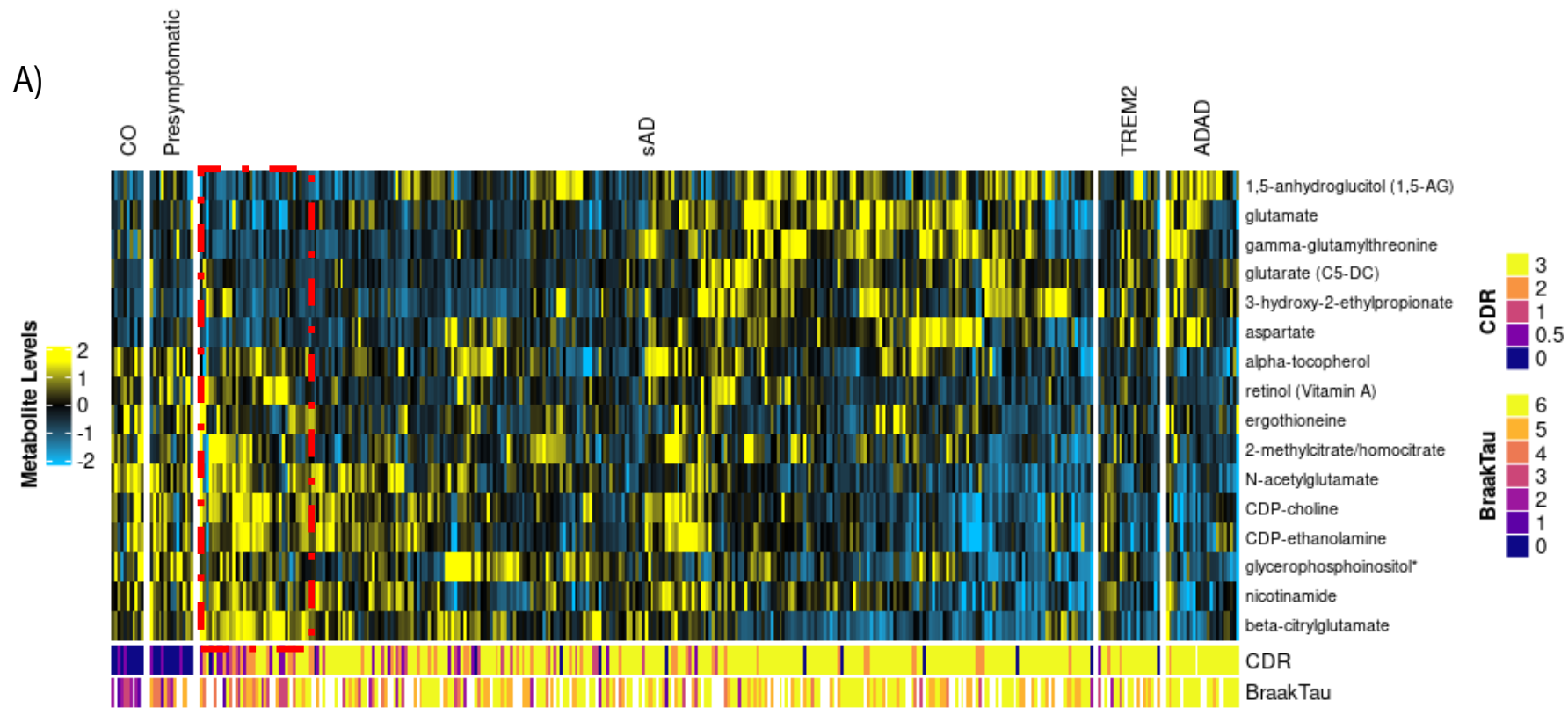

B)

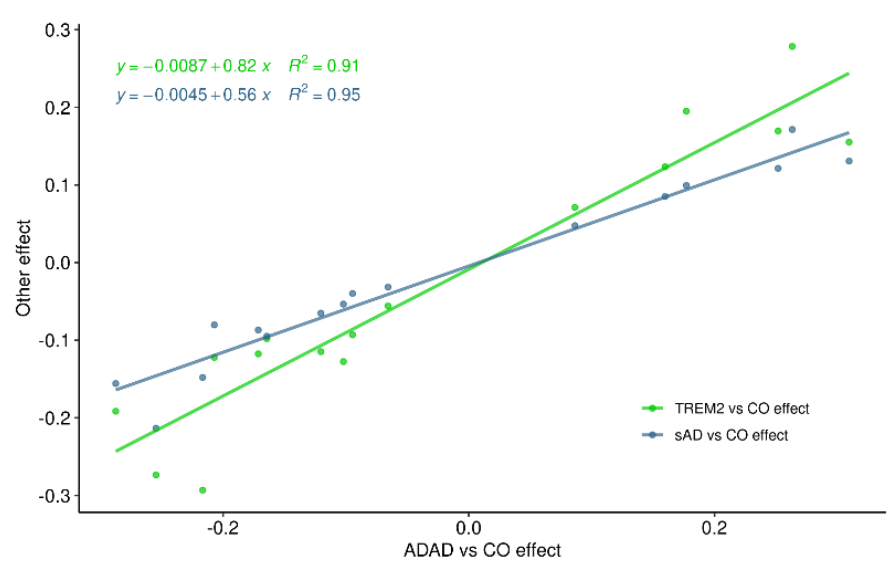

C)

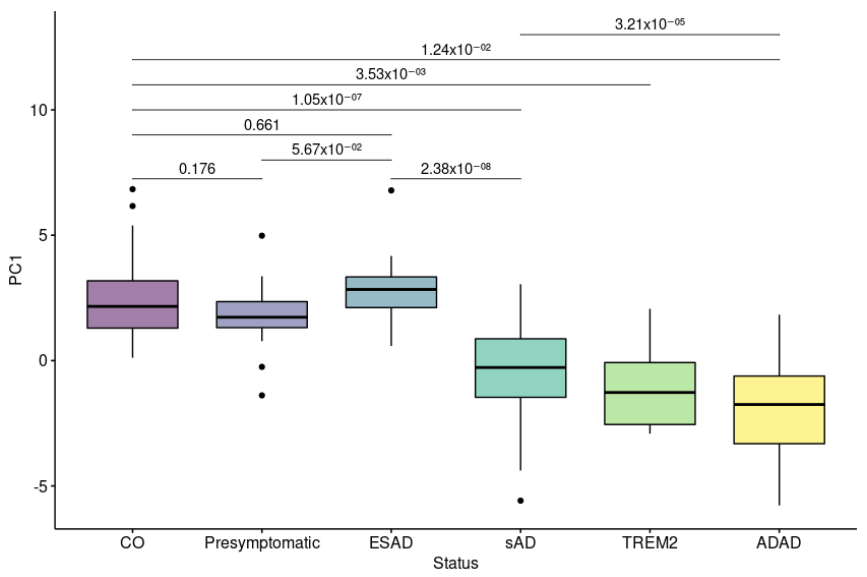

Figure 3. Metabolic profile consisting of 16 metabolites which passed FDR correction in ADAD vs $\mathrm{CO}$ and were at least nominally significant in SAD vs $C O$ and TREM2 vs CO. A) Heatmap showing relative abundance for each metabolite in the profile. Participants are divided by disease status group: healthy controls (CO), neuropathology but no cognitive impairment (Presymptomatic), sporadic AD (SAD), carriers of TREM2 risk variants (TREM2) and carriers of Mendelian mutations (ADAD). The 30 Early-Stage AD (ESAD) individuals identified by hierarchical clustering are indicated within the red box. Annotations show Clinical Dementia Rating and Braak scores for Tau accumulation. B) Comparison of effects for the 16 metabolites in each model. The $x$-axis shows the effect of each metabolite in the ADAD vs $C O$ model, while the $y$-axis shows the effects in the SAD vs $C O$ (blue) and TREM2 vs $C O$ (red) models. C) Boxplot showing distribution of the first principal component for the 16-metabolite profile among each of the status groups. 
medRxiv preprint doi: https://doi.org/10.1101/2021.11.04.21265941; this version posted November 30, 2021. The copyright holder for this preprint (which was not certified by peer review) is the author/funder, who has granted medRxiv a license to display the preprint in perpetuity.

It is made available under a CC-BY-NC-ND 4.0 International license .

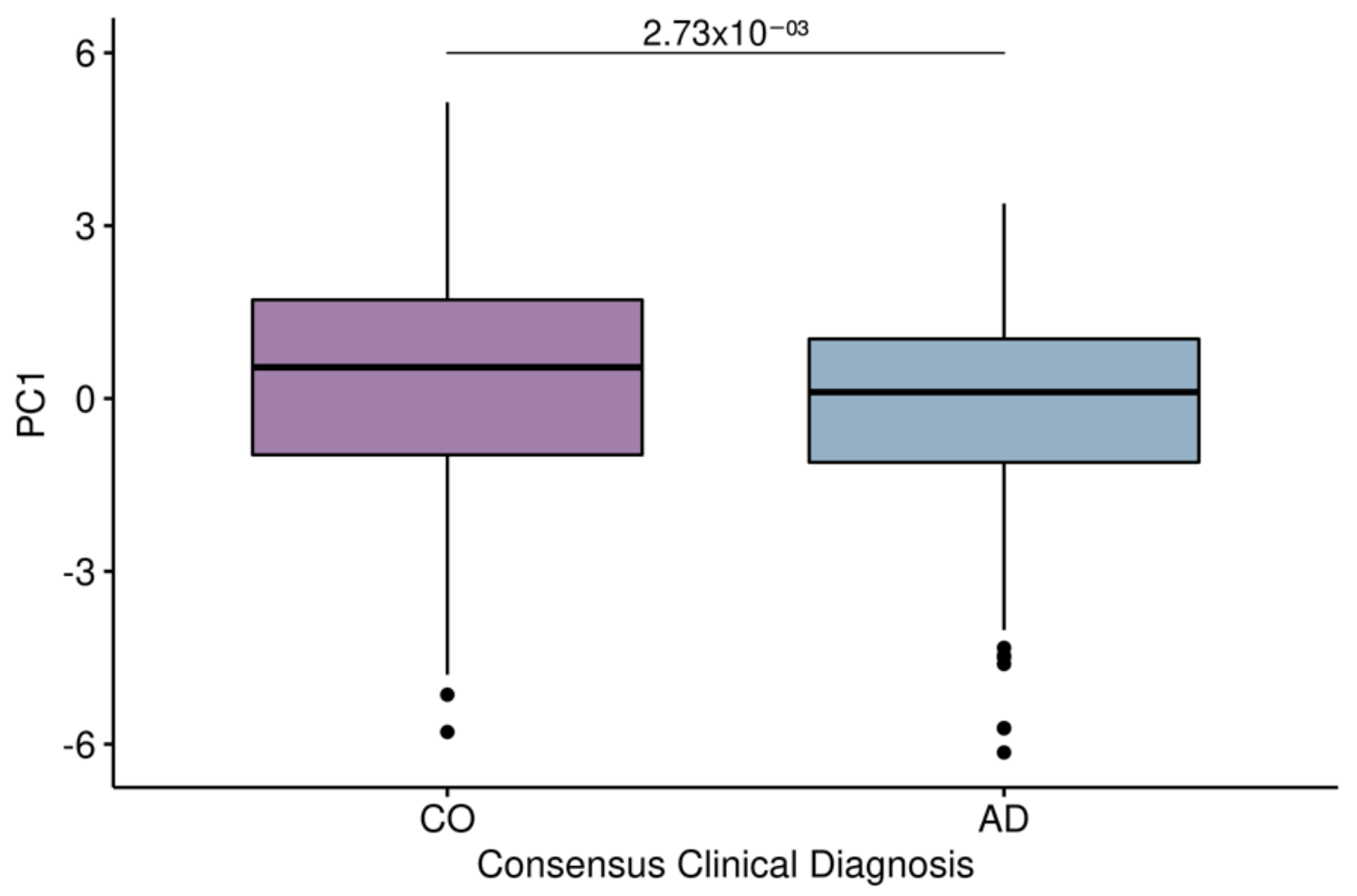

Figure 4. Distribution of metabolic eigenmetabolite profile between clinical diagnosis groups calculated with 15 metabolites on ROSMAP Metabolon data. 3-hydroxy-2-ethylpropionate was not included because it did not pass quality control in the ROSMAP dataset. 